\title{
Three-dimensional mesoscopic modeling of equiaxed dendritic solidification of a binary alloy ${ }^{\text {th }}$
}

\author{
Youssef Souhar $^{\mathrm{a}}$, Valerio Francesco De Felice ${ }^{\mathrm{a}}$, Christoph Beckermann ${ }^{\mathrm{b}}$, Hervé Combeau $^{\mathrm{a}}$, Miha Založnik ${ }^{\mathrm{a}}$ \\ ${ }^{a}$ Institut Jean Lamour, UMR 7198, CNRS - Université de Lorraine, F-54011 Nancy, France \\ ${ }^{b}$ Department of Mechanical and Industrial Engineering, University of Iowa, Iowa City, Iowa 52242, USA
}

\begin{abstract}
The mesoscopic envelope model is a recent multiscale model that is intended to bridge the gap between purely microscopic and macroscopic approaches for the study of dendritic solidification. It consists of the description of a dendritic grain by an envelope that links the active dendrite branches. The envelope growth is deduced from an analytical microscopic model of the dendrite tip growth kinetics matched to the numerical solution of the mesoscopic solute concentration field in the vicinity of the envelope. The branched dendritic structure inside the envelope is described in a volume-averaged sense by phase fractions and averaged solute concentrations. We present a careful quantitative analysis of the influence of numerical and model parameters on the accuracy of the model predictions. We further perform a validation study through comparisons of 3D simulations to experimental scaling laws giving the shape and the internal solid fraction of freely growing binary alloy dendrites and to analytical solutions for the primary dendrite tip speed. We provide generally valid guidelines for the calibration of the mesoscopic model, enabling reliable control of the accuracy of model predictions over a wide range of undercoolings. The model is applied to simulate strong solutal interactions in large ensembles of equiaxed grains. The potential for mesoscopic simulations to provide refined modeling of microstructures in volume-averaged macroscopic models via scale bridging is demonstrated.
\end{abstract}

Keywords: alloy solidification, dendritic growth, multiscale modeling, scale bridging, model validation

\section{Introduction}

Dendritic (treelike) crystals or grains are the most common growth form in solidification of metal alloys. Their growth is governed by an intricate interplay between diffusion or convection of heat and chemical species (solutes) and capillary effects. Furthermore, in castings the growth of dendritic crystals is influenced by adjacent grains. The grains can "feel" each other due to the overlap of thermal and solutal fields surrounding each growing grain. Analytical solutions of dendritic growth are limited to the description of a single isolated dendrite tip that grows by diffusion in an infinite, uniformly undercooled melt $[1,2]$.

Complex dendritic structures can be simulated directly by phase-field methods, which directly resolve the dendritic structure in detail but are computationally expensive. These and other microscopic methods thrived and matured in the last decade [3-5]. Phase-fields methods have become the most common approach to the numerical simulation of dendrite growth. However, because phase-field methods need

\footnotetext{
This is a post-peer-review, pre-copyedit version of an article published in Computational Materials Science: Y. Souhar, V.F. De Felice, C. Beckermann, H. Combeau, M. Zalonik, Three-dimensional mesoscopic modeling of equiaxed dendritic solidification of a binary alloy, Comput. Mater. Sci. 112 (2016) 304-317. The final authenticated version is available online at: http:// dx.doi.org/10.1016/j.commatsci.2015.10.028.

Email address: youssef@souhar.fr (Youssef Souhar)
}

a very fine mesh, computing and memory requirements are large. Most simulations are limited to the scale of a few dendrites, to two dimensions and purely diffusive conditions. Only recently simulations of large ensembles of grains in $3 \mathrm{D}$ have been reported [6, 7]. They required complex highperformance parallel computing algorithms and massive supercomputing resources.

Other common approaches are cellular-automaton and volume-averaged models [8-13] that can simulate the growth of multiple dendrites on the scale of an entire casting but at the expense of simplifications. These methods are not able to predict accurate grain shapes and rely on very approximate relations for modeling grain interactions.

A simulation tool for dendritic solidification that is intended to bridge the gap between purely microscopic and macroscopic approaches is given by the so-called mesoscopic solidification model of Steinbach, Beckermann and coworkers [14-16]. This model relies on the description of a dendritic grain by its envelope, which is a smooth surface connecting all of the actively growing dendrite tips. An example of interacting three-dimensional equiaxed dendritic grain envelopes predicted by the mesoscopic model is shown in Fig. 1. The driving force for the envelope growth is obtained as a function of the temperature or solute concentration in the liquid at a certain distance ahead of the envelope. As shown in Fig. 2, this distance is denoted as the stagnant film thickness $\delta$ and is the principal model parameter. This model has 


\section{Nomenclature}

\section{Greek Letters}

$\Gamma \quad$ Gibbs-Thompson coefficient

$\delta \quad$ Stagnant film thickness (distance between the envelope and the confocal envelope)

$\delta_{\text {env }} \quad$ Distance to the envelope

$\delta_{1} \quad$ Volume-averaged diffusion length at the envelope

$\theta \quad$ Angle between the envelope normal and the tip growth direction

$\sigma^{*} \quad$ Tip selection parameter

$\tau \quad$ Dimensionless time scaled by $D_{1} / V_{\mathrm{LGK}}^{2}$

$\tau_{\mathrm{d}} \quad$ Dimensionless time of departure from free growth

$\tau_{\Psi \min } \quad$ Dimensionless time of minimum sphericity

$\phi \quad$ Phase indicator field

$\Psi \quad$ Average envelope sphericity

$\Omega \quad$ Supersaturation

$\Omega_{0} \quad$ Initial supersaturation

$\Omega_{\delta} \quad$ Supersaturation at the confocal envelope

$\Omega_{\infty} \quad$ Supersaturation at infinity

\section{Latin letters}

$A_{1}, A_{2} \quad$ Amplification factors in the dendrite scaling laws

$b, b_{\lim } \quad$ Stabilization parameter and its stability limit

C Solute concentration

$\left\langle C_{1}\right\rangle^{\mathrm{e}} \quad$ Average solute concentration in the extradendritic liquid

$\mathrm{Co}_{\phi} \quad$ Courant number of the phase indicator field

D Solute diffusion coefficient

$d_{0} \quad$ Capillary length

$\bar{d}_{\mathrm{cc}} \quad$ Mean distance between grain centers

$F \quad$ Projection area of the solid phase

$\mathrm{Fo}_{\Delta x} \quad$ Grid Fourier number

$g \quad$ Volume fraction

$k_{\mathrm{p}} \quad$ Equilibrium solute partition coefficient $l_{\text {diff }} \quad$ Theoretical (LGK) solutal diffusion length at the primary tip at the initial supersaturation

$m_{\mathrm{L}} \quad$ Slope of the liquidus line

$n_{1}, n_{2} \quad$ Exponents in the dendrite scaling laws

$\vec{n} \quad$ Outward drawn normal of the envelope surface

$N_{V}^{\text {ldiff }} \quad$ Dimensionless grain density

Pe Péclet number

$R \quad$ Radius

$S_{\mathrm{V}, \text { env }} \quad$ Volume averaged specific envelope surface area

$T \quad$ Temperature

$T_{\mathrm{f}} \quad$ Melting temperature of the pure solvent

$t \quad$ Time

$V, \vec{V} \quad$ Speed, velocity

$W \quad$ Characteristic width of the hyperbolic-tangent profile

$X_{\text {act }} \quad$ Width of the envelope formed by active sidebranches

$\vec{x}_{\delta} \quad$ Position of the confocal envelope point

$x, y, z \quad$ Cartesian coordinates

$\Delta x, \Delta t$ grid spacing, timestep

Subscripts

e Envelope

env Envelope surface

LGK Theoretical LGK primary dendrite tip

1 Liquid phase

$s \quad$ Solid phase

tip Dendrite tip

Superscripts

* $\quad$ Solid-liquid interface

Dimensionless quantity

Special functions

Iv Ivantsov function : $\operatorname{Iv}(\mathrm{Pe})=\operatorname{Pe} \exp (\mathrm{Pe}) \mathrm{E}_{1}(\mathrm{Pe})$ been shown to provide physically realistic results for both equiaxed $[14,15]$ and columnar $[16,17]$ dendritic growth.

Steinbach et al. [14] applied the mesoscopic model to the thermally driven growth of dendritic grains into a supercooled melt of a pure substance. They validated the predictions of the model for the case of a single grain growing into an essentially infinite melt through comparisons with previously obtained scaling laws for the grain envelope shape and the internal solid fraction, derived from microgravity experimental data [19-22]. Later they investigated the transient interactions between equiaxed grains of a pure substance and validated some of their results with phase-field calculations [15]. More recently, Delaleau et al. [16] extended the mesoscopic model to the solidification of a binary alloy with a prescribed temperature field. They applied the model to simulate the columnar dendritic microstructures observed in in-situ synchrotron X-ray imaging experiments [23, 24]. Relatively good agreement was found between the predicted and measured dendrite envelope shapes, solid fractions and solute concentration fields.

These studies have shown the potential of the mesoscopic model for accurate prediction of dendrite envelope shapes and grain interactions at a computational cost that is up to several orders of magnitude smaller than that of phase-field methods [17]. An interesting future application of this model is the upscaling from mesoscopic simulations to volumeaveraged macroscopic models, in order to provide laws of microstructure growth dynamics that account for interactions in large ensembles of grains. This can be done, for example, by simulations of large ensembles of grains across wide parameter ranges and by subsequent averaging of these simulations. However, on the way to such a wide application 


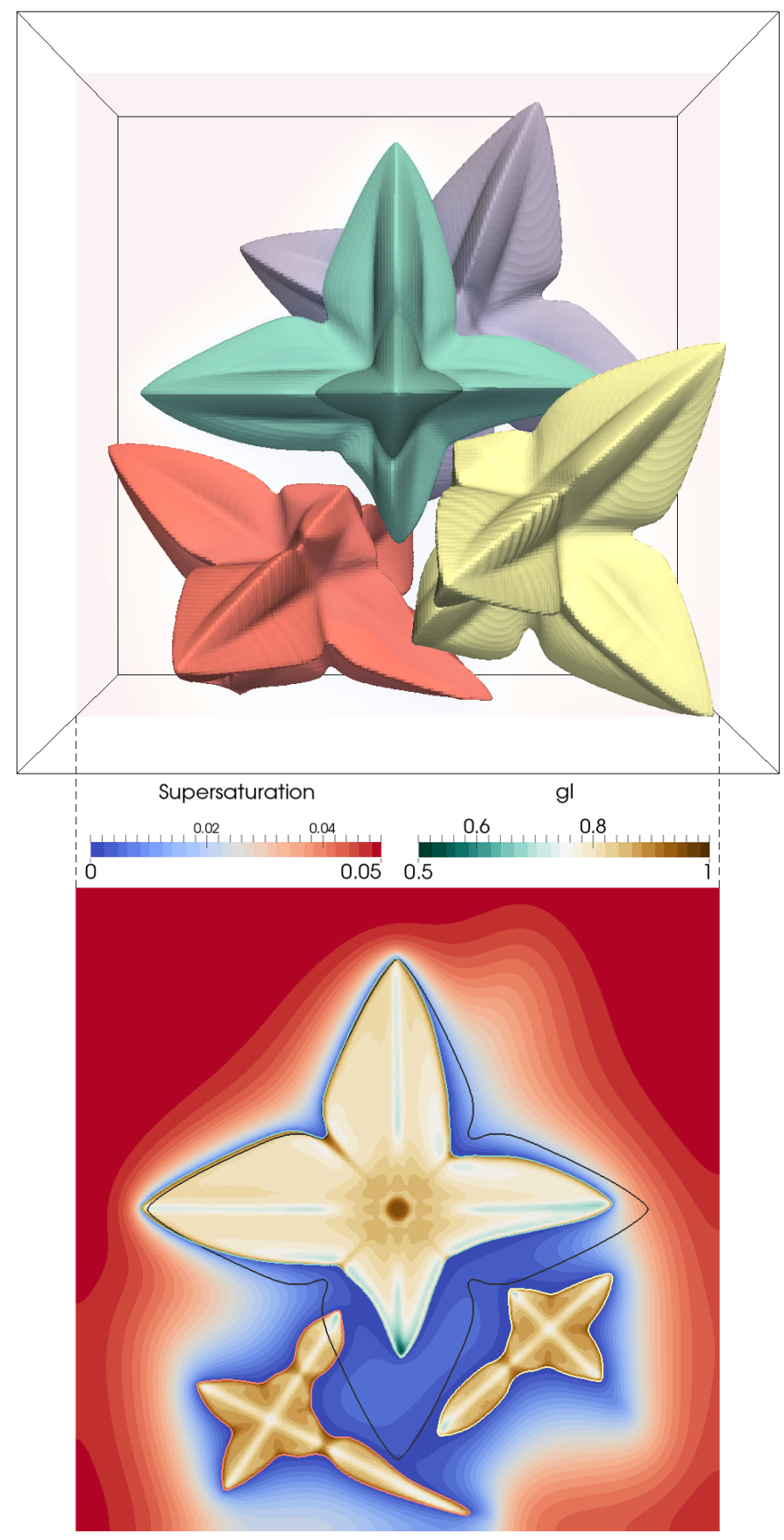

Fig. 1 Example of results of a simulation with the mesoscopic model. Top: four interacting equiaxed grain envelopes growing in an enclosure. Bottom: a slice across the enclosure at mid-depth (shaded area in the top image). The liquid fraction field $\left(g_{1}\right)$ is shown in the interior of the envelopes and the supersaturation field in the liquid outside the envelopes. The black line is the contour of the envelope of a free (unconfined) equiaxed grain aligned with the green grain and growing at the same initial supersaturation of $\Omega_{0}=0.05$. Note that the core of the central dendrite shows a zone with $g_{\mathrm{s}}=\Omega_{0}$ which is a trace of the initial spherical nucleus.

of this model, a comprehensive investigation of the accuracy of the model predictions, particularly of the dependence of the simulations on the model and numerical parameters, is still necessary. This has not been systematically addressed in previous studies. In the present paper we perform a care-

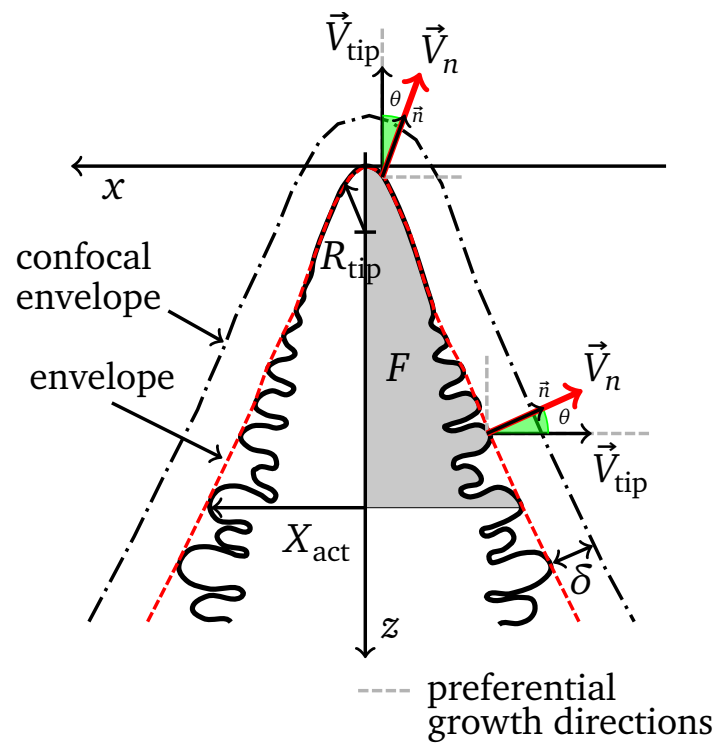

Fig. 2 Schematic illustration of the main principles of the mesoscopic model: the envelope, the confocal envelope, and the stagnant film thickness, $\delta$. The envelope propagation velocity, $\vec{V}_{n}$, is obtained by the projection of the tip velocity, $\vec{V}_{\text {tip }}$, from the preferential growth direction forming the smallest angle $\theta$ with the envelope normal, $\vec{n}$. The parameters and scales of equiaxed dendrites measured in the experiments used for validation [18] are also shown: the width of the envelope formed by the active sidebranches, $X_{\text {act }}$, the projection area of the solid, $F$, and the primary tip radius, $R_{\text {tip }}$.

ful quantitative analysis of the influence of numerical and model parameters on the accuracy of the model predictions. We also validate the model for free dendritic growth in a binary alloy over a wide range of undercoolings. This is accomplished through comparisons of $3 \mathrm{D}$ simulations to recently published experimental scaling laws giving the shape and the internal solid fraction of freely growing binary alloy dendrites [18] and to classical analytical solutions for the primary dendrite tip speed. We provide generally valid guidelines for the calibration of the numerical and model parameters of the mesoscopic model, enabling reliable control of the accuracy of model predictions over a wide range of undercoolings. Finally, we apply the model to large ensembles of equiaxed grains growing in the presence of strong solutal interactions. The potential for mesoscopic simulations to provide refined modeling of microstructures in volume-averaged macroscopic models via scale bridging is demonstrated using these examples.

\section{Description of the model}

The core idea of the mesoscopic envelope model is the description of a dendritic grain by its envelope - a virtual smooth surface that links the tips of the actively growing dendrite branches (see Fig. 2). The growth velocity of the envelope can thus be calculated from the velocities of the dendrite tips. The growth of the dendrite tips is controlled by the solute flux that they eject into their surroundings and is therefore determined by the local supersaturation of the liquid in the vicinity of the envelope. The branched dendritic structure 
inside the envelope is only implied and its details are not resolved; the interior of the envelope is instead described in a volume-averaged sense by a phase-fraction field, as shown in Fig. 1. The phase solute concentrations are also described in a volume-averaged sense. The phase change that determines the evolution of the structure, i.e. of the phase fraction field, inside the growing envelope is controlled by the exchange of solute with the surroundings of the grain. The transport of solute at the mesoscopic scale is described by volume-averaged transport equations.

The model equations are given in dimensionless form in order to provide better insight into the role played by the control parameters. Scalings related to the free Ivantsov dendrite tip are most convenient for nondimensionalization. They are obtained by a simplified version of the LiptonGlicksman-Kurz (LGK) tip model [25] that neglects thermal and capillary undercooling and accounts only for the solutal contribution. The free tip grows at a supersaturation of $\Omega_{\infty}=\left(C_{1}^{*}-C_{\infty}\right) /\left(C_{1}^{*}\left(1-k_{\mathrm{p}}\right)\right)$, where $C_{1}^{*}$ is the liquid concentration at the solid-liquid interface of the tip growing at the temperature $T=T_{\mathrm{f}}+m_{\mathrm{L}} C_{1}^{*}, C_{\infty}$ is the liquid concentration at infinity, $k_{\mathrm{p}}$ is the equilibrium solid-liquid solute partition coefficient, $T_{\mathrm{f}}$ is the melting temperature of the pure solvent, and $m_{\mathrm{L}}$ is the liquidus slope. The inverse Ivantsov function provides the growth Péclet number of the tip, $\mathrm{Pe}_{\mathrm{LGK}}=$ $R_{\mathrm{LGK}} V_{\mathrm{LGK}} /\left(2 D_{1}\right)$, as a function of the supersaturation via the relation $\operatorname{Pe}_{\mathrm{LGK}}=\operatorname{Iv}^{-1}\left(\Omega_{\infty}\right)$, where $\operatorname{Iv}(x)=x \exp (x) \mathrm{E}_{1}(x)$. The tip selection criterion is given by $R_{\mathrm{LGK}}^{2} V_{\mathrm{LGK}}=d_{0} D_{1} / \sigma^{*}$, where $d_{0}=\Gamma /\left(m_{\mathrm{L}} C_{1}^{*}\left(k_{\mathrm{p}}-1\right)\right)$ is the solutal capillary length, $\sigma^{*}$ is the selection constant, and $\Gamma$ is the Gibbs-Thomson coefficient. These relations give the free-tip curvature radius, $R_{\mathrm{LGK}}=d_{0} /\left(2 \sigma^{*} \mathrm{Pe}_{\mathrm{LGK}}\right)$, which is used as the length scale, and the free-tip speed, $V_{\mathrm{LGK}}=4 \sigma^{*} D_{1} \mathrm{Pe}_{\mathrm{LGK}}^{2} / d_{0}$, which is used as the velocity scale. Time is scaled by the square of the tip radius over the solute mass diffusivity, $R_{\mathrm{LGK}}^{2} / D_{1}$, and the concentration scale is $C_{1}^{*}\left(1-k_{\mathrm{p}}\right)$. The dimensionless position vector, speed, time, and solute concentration are then given by $\tilde{\vec{x}}=\vec{x} / R_{\mathrm{LGK}}, \tilde{V}=V / V_{\mathrm{LGK}}, \tilde{t}=t D_{1} / R_{\mathrm{LGK}}^{2}$, and $\Omega=\left(C_{1}^{*}-C\right) /\left(C_{1}^{*}\left(1-k_{\mathrm{p}}\right)\right)$, respectively.

\subsection{Dendrite tip kinetics}

The local growth speed of the envelope is given by the kinetics of the dendrite tips behind it. Their growth Péclet number, $\mathrm{Pe}_{\text {tip }}$, is obtained from a modified Ivantsov solution that is written in terms of the supersaturation, $\Omega_{\delta}$, at a finite distance $\delta$ from the tip [26]:

$$
\Omega_{\delta}=\mathrm{Pe}_{\text {tip }} \exp \left(\mathrm{Pe}_{\text {tip }}\right)\left[\mathrm{E}_{1}\left(\mathrm{Pe}_{\text {tip }}\right)-\mathrm{E}_{1}\left(\mathrm{Pe}_{\text {tip }}\left[1+\frac{2 \delta}{R_{\text {tip }}}\right]\right)\right]
$$

Equation (1) is also called the stagnant-film formulation of the Ivantsov solution. In the mesoscopic model, the supersaturation $\Omega_{\delta}$ is obtained from the numerically resolved concentration field in the liquid around the grain envelope. This corresponds to a matching of the locally valid tip solution to the solution of the mesoscopically valid solute transport equation at a distance $\delta$ from the envelope (on a surface called the confocal envelope, Fig. 2). In this way the tip kinetics and the mesoscale solutal interactions are fully coupled without resolving the structure of individual dendrite branches.

Equation (1) is supplemented by the tip selection criterion, which can be written in dimensionless form as $\tilde{R}_{\text {tip }}^{2} \tilde{V}_{\text {tip }}=1$. Then, the dimensionless tip speed is given by:

$$
\tilde{V}_{\text {tip }}=\left(\frac{\mathrm{Pe}_{\text {tip }}}{\mathrm{Pe}_{\mathrm{LGK}}}\right)^{2} \text {. }
$$

Equations (1) and (2) together give the local tip speed at any point on the grain envelope. The stagnant film thickness, $\delta$, is a model parameter. Its choice is not clear a priori and, as we will show, $\delta$ has an influence on the results (primary tip speed, envelope shape, etc.).

\subsection{Envelope propagation with the phase-field equation}

One of the main assumptions of the mesoscopic model is that the preferential tip growth directions are known. They must be prescribed, since the physics underlying the selection of the growth direction is not included in the model. For example, a typical cubic crystal dendrite can be approximated by six possible growth directions that are perpendicular to each other. The normal envelope growth velocity, $\vec{V}_{n}$, is then calculated from the local tip speed, $V_{\text {tip }}$, by the relation

$$
\tilde{\vec{V}}_{n}=\tilde{V}_{\text {tip }} \vec{n} \cos \theta
$$

where $\theta$ is the angle between the outward drawn normal to the envelope, $\vec{n}$, and the preferential growth direction that forms the smallest angle with the normal, as shown in Fig. 2.

As in previous work [14-16], we use the phase-field sharpinterface capturing method [27] to propagate the envelope on a numerical mesh. In this method the tracked front is given by the level set of a continuous indicator field $\phi$. The transition of $\phi$ from 1 to 0 in the vicinity of the front follows a hyperbolic tangent profile given by the kernel function [28]

$$
\phi(n)=\frac{1}{2}\left[1-\tanh \left(\frac{n}{2 W}\right)\right],
$$

where $n$ is the distance from the center of the profile. The phase-field equation that is used to propagate the field ensures that the profile is self preserving and retains its shape and its characteristic width $W$. In our case the envelope is defined by the level set of $\phi=\phi_{\text {env }}=0.95$. This value can be chosen arbitrarily; however, it affects the error associated with the approximation of a curved front by the phase field. For the method to work accurately, $\phi$ profiles should not overlap in curved parts of the surface. The largest overlap is determined by the smallest radius of curvature of the front, $R_{\text {env,min }}$, and by the width of the kernel function, $W$, which varies from 1 to 0 over a distance of approximately $6 W$. If $\phi_{\text {env }}=0.5$ is used, $W$ should be chosen such that $W \leq R_{\text {env,min }} / 6$ in order to describe the shape with sufficient accuracy [27]. If $\phi_{\text {env }}=0.95$ is used, a kernel function width of $W \leq R_{\text {env,min }} / 3.1$ is sufficiently small to accurately describe the convexly curved parts of the front. However, in concave parts, only curvatures with a radius larger than $8.9 \mathrm{~W}$ can be 
accurately described in this case. Generally, we seek to set $W$ as large as possible, because the grid spacing must meet the requirement $\Delta x \leq W / \sqrt{2}$ [27] in order to describe the hyperbolic tangent transition with sufficient accuracy. By using a larger $W$ the grid spacing can be increased and the computational cost is thereby decreased. The choice of $\phi_{\text {env }}=0.95$ was motivated by the fact that we studied primarily the tip of the envelope of the primary arm, where the convex curvatures are most critical. As long as the $\phi$-profile is well conserved and the smallest radius of curvature is compatible with the studied case, there is no particular sensitivity of the model to the choice of $\phi_{\text {env }}$.

The phase-field equation for the propagation of the indicator function $\phi$ is [28]:

$$
\begin{aligned}
& \frac{\partial \phi}{\partial t}+V_{n} \vec{n} \cdot \nabla \phi= \\
& -b \underbrace{\left[\nabla^{2} \phi-\frac{\phi(1-\phi)(1-2 \phi)}{W^{2}}-|\nabla \phi| \nabla \cdot\left(-\frac{\nabla \phi}{|\nabla \phi|}\right)\right]}_{\text {stabilization term }},
\end{aligned}
$$

The term on the right hand side of the equation is a stabilization term that ensures that the phase-field $\phi$ retains the hyperbolic tangent profile. The coefficient $b$ is a relaxation factor. It has to be chosen appropriately to minimize the error and to ensure the stability of the method [27]. The choice of $b$ in the context of the mesoscopic model is discussed in Section 3.

\subsection{Transport equations}

The solute transport at the mesoscopic scale is described by volume averaged equations [29] that are valid in the whole domain, i.e., both inside and outside the envelopes. The solid and liquid phases are assumed to be stationary. Solidification inside the envelope is modeled using the Scheil assumptions: thermodynamic equilibrium at the solid-liquid interface, negligible diffusion in the solid and perfect diffusion in the liquid. This implies that the supersaturation of the liquid inside the envelope is zero and the dimensionless concentration of the solid at the solid-liquid interface is $\Omega_{\mathrm{s}}^{*}=1$. These assumptions lead to the following simplified dimensionless conservation equation for the solute in the liquid phase that describes isothermal solidification at a constant temperature, such as will be treated in our simulations:

$$
g_{1} \frac{\partial \Omega_{1}}{\partial \tilde{t}}=\tilde{\nabla} \cdot\left(g_{1} \tilde{\nabla} \Omega_{1}\right)+\frac{\partial g_{1}}{\partial \tilde{t}}
$$

Outside the envelope the material is fully liquid,

$$
g_{1}=1 ; \phi<\phi_{\text {env }}
$$

and Eq. (6) reduces to a single phase diffusion equation. Inside the envelope, the liquid is in thermodynamic equilibrium, such that:

$$
\Omega_{1}=0 \quad ; \quad \phi \geq \phi_{\mathrm{env}}
$$

With $\Omega_{1}$ known, Eq. (6) gives the evolution of the liquid fraction inside the envelope. In the particular case of isothermal growth at a constant temperature, $\Omega_{1}$ in the interior of the envelope is uniform and constant and Eq. (6) therefore reduces to $\partial g_{1} / \partial \tilde{t}=0$. Phase change in this case only occurs at the envelope front, where a solid fraction $g_{\text {s,env }}$ forms due to the solute rejected from the envelope into the surrounding liquid. It is given by the solute flux balance at the envelope:

$$
g_{\mathrm{s}, \mathrm{env}} \tilde{V}_{n}=\left.\frac{\partial \Omega_{1}}{\partial \tilde{n}}\right|_{\mathrm{env}},
$$

During the propagation of the envelope, the concentration gradient at the envelope varies in space and time. As a trace of the varying conditions, a certain solid fraction distribution develops inside the envelope, as can be seen in Fig. 1. Note that we do not solve Eq. (9) explicitly; it is merely a consequence of solving Eqs. (6-8). Note also that solving this equation system is equivalent to: (i) setting $\Omega_{1}=0$ and then solving Eq. (6) for $g_{1}$ inside the envelope; and (ii) setting $g_{1}=1$ and solving Eq. (6) for $\Omega_{1}$ outside the envelope.

\section{Numerical implementation}

We solve the model, consisting of the system of Eqs. (1-3) and (5-8), by the following sequence of steps in each time increment:

1. Determination of the supersaturation at the confocal envelope, $\Omega_{\delta}$, for each grid element within the region around the envelope front.

2. Calculation of the envelope propagation velocity field, $\vec{V}_{n}$, by local solution of the dendrite tip model (Eqs. (13)).

3. Solution of the phase-field equation (5) by an explicit time-stepping scheme.

4. Solution of the conservation equations for liquid fraction and for solute mass in the liquid (Eqs. (6-8)) by an operator-splitting algorithm with implicit time stepping [30].

The transport equations were solved with the finite volume method using first-order Euler schemes for time stepping. The gradient of the phase-field was discretized by a fourth-order accurate scheme using least-squares weighting. The Laplacian terms in the phase-field and solute equation (6) were evaluated using a fourth-order central scheme. The divergence in the stabilization term of Eq. (5) was discretized using a second-order scheme.

The computer code CrystalFOAM ${ }^{\circledR}$ was developed on the OpenFOAM $^{\circledR}$ platform. It uses 3D finite-volume solvers, parallelized by domain decomposition and employing Open MPI for parallel computation on distributed memory systems, achieving good scalability. Most development efforts went into the development of the algorithm for the determination of the supersaturation at the confocal envelope. 


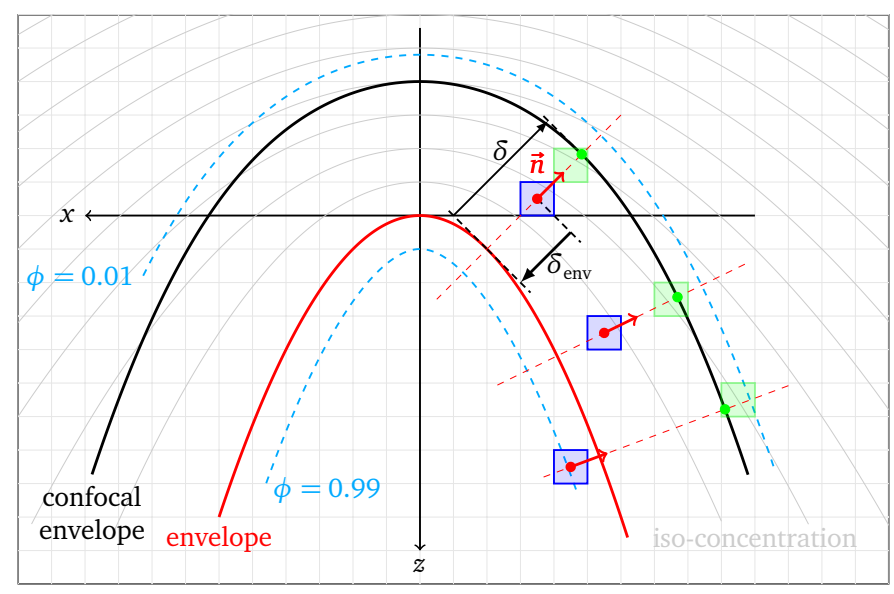

Fig. 3 Schematic illustration of the method used to compute the envelope propagation velocity, $\vec{V}_{n}$, for all grid cells within the transition region of the indicator field: $0.01<\phi<0.99$ (blue cells). The supersaturation $\Omega_{\delta}$ needed for this is the supersaturation of the liquid in the corresponding green dots, i.e. at the intersection of the confocal envelope and the line of the envelope normal vector running through the center of the blue cell (red dot). $\Omega_{\delta}$ is calculated by interpolation across the neighborhood of the green cell.

\subsection{Determination of the envelope propagation velocity}

To propagate the phase field that describes the position of the envelope, the envelope growth velocity has to be given in the form of a corresponding propagation velocity field $\vec{V}_{n}$ in the whole domain. In practice, it is sufficient to determine the propagation velocity in a relatively narrow band around the transition region of the field $\phi$ in the vicinity of the envelope. We used a band in the interval $0.01<\phi<0.99$. In order to preserve the hyperbolic tangent shape in $\phi$, the propagation velocity should be constant across a cross-section perpendicular to the envelope, i.e. in the direction of the envelope normal. Since the elements of the computational grid are generally not aligned along this direction, it is not possible to obtain the propagation velocity across the whole width of the transition at once. The propagation velocity has to be actually calculated for every grid cell within the transition region. Examples are shown in Fig. 3 as blue cells. This is done by solving Eqs. (1) and (2) for each of these cells. The supersaturation at the confocal envelope in the direction normal to the envelope $\Omega_{\delta}$ is needed for this.

In order to determine $\Omega_{\delta}$, the distance and the direction from the grid cell center, $\vec{x}$, to the corresponding point at the confocal envelope, $\vec{x}_{\delta}$, need to be determined first. The direction is given by the envelope normal vector, $\vec{n}$, that goes through the given grid cell center, $\vec{x}$. This vector is estimated as $\vec{n}=-\nabla \phi /|\nabla \phi|$, where $\nabla \phi$ is the local gradient in point $\vec{x}$, calculated by a discrete finite-volume approximation. The distance is given by $\delta-\delta_{\text {env }}$, where $\delta_{\text {env }}$ is the distance from point $\vec{x}$ to the envelope, which lies at $\phi_{\text {env }}=0.95$, along the same (normal) direction. A simple method for the calculation of $\delta_{\text {env }}$ is by the inverse of the hyperbolic tangent function:

$$
\delta_{\mathrm{env}}=W \ln \left(\frac{\left(1-\phi_{\mathrm{env}}\right) \phi(\vec{x})}{\phi_{\mathrm{env}}(1-\phi(\vec{x}))}\right) .
$$

This method is based on the assumption that the transition of the phase field closely follows the hyperbolic tangent profile. In practice, the phase field often slightly deforms, despite the stabilization terms. This is most pronounced in parts with high curvature, such as the tip of the dendrite envelope. If the shape of the transition deviates from the hyperbolic tangent, the distance $\delta_{\text {env }}$ given by the inverse kernel function (Eq. (10)) is not accurate any more. This leads to an error in the determination of the position of the confocal envelope point, $\vec{x}_{\delta}=\vec{x}+\left(\delta-\delta_{\text {env }}\right) \vec{n}$, and consequently to an error in the confocal envelope supersaturation, $\Omega_{\delta}=\Omega_{1}\left(\vec{x}_{\delta}\right)$. This is illustrated in Fig. 4, which shows the profiles of the phasefield $\phi$ and of the supersaturation $\Omega_{1}$ of the liquid along the axis of the primary tip of an envelope. We can see that the profile of $\phi$ is spread to a somewhat wider shape than the theoretical hyperbolic tangent that it intersects at the envelope (at $\phi_{\text {env }}=0.95$ ). As a consequence, the position of the confocal envelope estimated by the inverse kernel function is estimated to be at a distance from the envelope that is larger than $\delta$. Since the supersaturation increases with the distance from the envelope, the confocal envelope supersaturation, $\Omega_{\delta}$, is thus overestimated. The sensitivity to this error is generally higher if the chosen stagnant film thickness, $\delta$, is small and the confocal envelope is thus located close to the envelope in a region of high concentration gradients. We can also see that the error is not uniform across the width of the transition profile. The inverse kernel function method is accurate at the envelope $\left(\phi=\phi_{\text {env }}\right)$ and the error increases with the distance from the envelope. The consequence of the nonuniform error is a nonuniform normal envelope speed $V_{n}$, which is calculated from the supersaturation $\Omega_{\delta}$ for each cell within the transition region. Since an outward oriented spread of the $\phi$ transition induces a higher outward oriented propagation velocity, which increases the spread, this error is self-reinforcing. The final consequence is an inaccurate envelope propagation velocity.

The spread of the transition is nevertheless limited by the stabilization term of the phase-field propagation equation. A higher value of the stabilization coefficient, $b$, in Eq. (5) generally keeps the transition more compact and provides better accuracy. The maximum value of $b$ is however limited by the stability of the solution scheme. Even by setting $b$ close to the stability limit does not sufficiently reduce the errors of the envelope growth velocity. In order to avoid these errors, we used a front reconstruction method for the determination of the distance to the envelope. In this method the envelope is described by marker points distributed over the surface defined by the level set of $\phi=\phi_{\text {env }}$. This reconstruction is done at each timestep, using a marching tetrahedra method implemented in OpenFOAM ${ }^{\circledR}$ [31]. The marker points have a higher density than the finite-volume mesh, the envelope is thus reconstructed with good accuracy. An example of the reconstruction is shown in Fig. 5. The perpendicular distance of a grid cell point from the envelope is determined by the distance to the closest marker point projected onto the local unit gradient vector $\nabla \phi /|\nabla \phi|$. This method is general if the grid cell is on the convex side of the curved envelope surface. If it is on the concave side, the method works as 


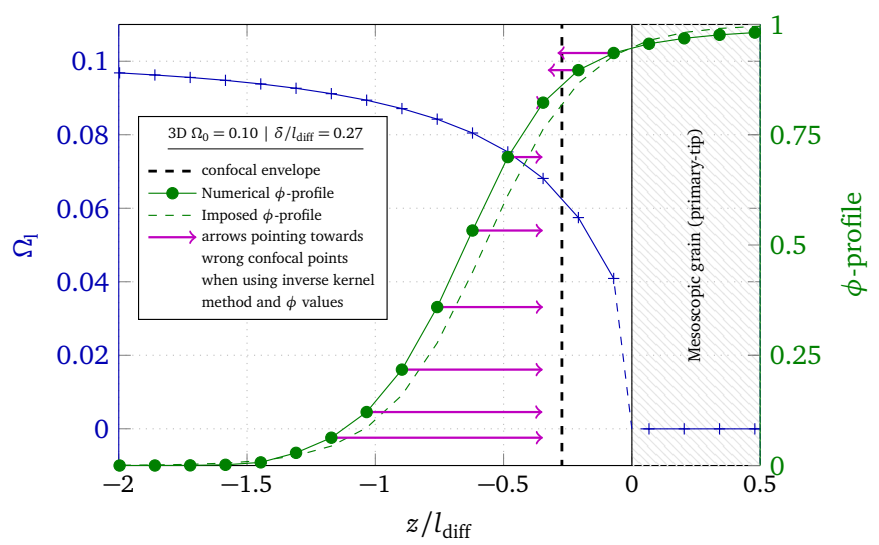

Fig. 4 Illustration of the error induced by the use of the inverse kernel function (Eq. (10)) for the determination of the distance to the confocal envelope. The deviation of the profile of the indicator field $\phi$ (solid green line) from the shape of the hyperbolic tangent kernel function (dashed green line intersecting the solid line at the envelope $\phi_{\text {env }}=0.95$ ) leads to an erroneous determination of the distance (arrows) between the grid cell centers (green dots) and the confocal envelope (dashed line). This type of error tends to estimate the position of the confocal envelope to be at a distance from the envelope larger than $\delta$, thus overestimating the supersaturation $\Omega_{\delta}$. The erroneous $\Omega_{\delta}$ is the supersaturation (blue line) at the positions of the arrowheads. The correct $\Omega_{\delta}$ is the supersaturation at the confocal envelope.

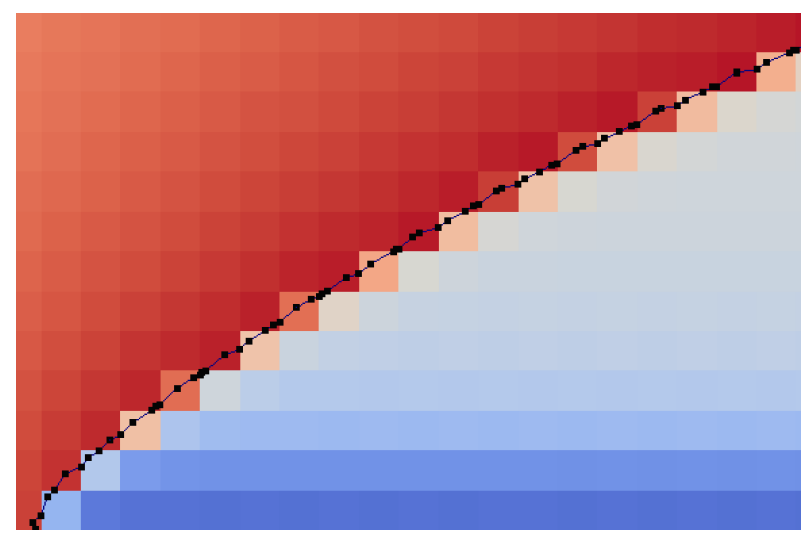

Fig. 5 Envelope markers determined at each timestep by the marching tetrahedra algorithm [31]. The squares correspond to the finite volume elements.

long as the distance to the envelope is smaller than the local curvature of the envelope. The front reconstruction method substantially improves the accuracy of the determination of the envelope growth velocity, especially for small stagnant film thicknesses.

Once the position of the corresponding confocal point, $\vec{x}_{\delta}=\vec{x}+\delta_{\text {ce }} \vec{n}$, is determined, the liquid supersaturation at this point, $\Omega_{\delta}$, is determined by a linear interpolation across the neighborhood of the cell containing $\vec{x}_{\delta}$ (green cells in Fig. 3). The supersaturation at the envelope, $\Omega_{\text {env }}$, can in general be determined by the same procedure, but in the isothermal case treated here it was known a priori. Knowing both the supersaturation at the envelope and at the confocal envelope, the supersaturation $\Omega_{\delta}$ is computed. Eqs. (1-2) are then solved by a hybrid Newton-bisection method, giving the tip speed. Finally, the projection onto the envelope (Eq. (3)) gives the envelope propagation velocity, $\vec{V}_{n}$.

\subsection{Numerical parameters and grid convergence}

In this section we will discuss the choice of the numerical parameters of the present implementation of the mesoscopic model. The solution method is controlled by two parameters of the phase-field interface capturing method, the kernel function width, $W$, and the stability coefficient, $b$, and by the spatial and temporal discretization steps, $\Delta x$ and $\Delta t$. The stagnant film thickness, $\delta$, is a model parameter with a physical meaning and will be discussed separately.

Following the recommendations in [27], the characteristic width of the kernel function of Eq. (4) is chosen such that $W=\sqrt{2} \Delta x$ in order to accurately resolve the hyperbolic tangent profile. Thus, the $\phi$ profile varies from 1 to 0 over a distance of approximately $6 W \approx 8 \Delta x$. The radii of the spherical envelope "nuclei" that were introduced to initialize the grains, were taken slightly larger than $6 \mathrm{~W}$ in order to ensure good accuracy from the onset of the simulation.

According to Sun and Beckermann [27] the accuracy of the interface capturing method increases with an increasing stabilization parameter $b$, up to a stability limit $b_{\text {lim }}$ that depends on the front velocity and on the spatial and temporal discretization. The stabilization parameter $b$ was set adaptively in time, as a fraction of the current stability limit. At timestep $n$ it was set to:

$$
\begin{aligned}
b^{(n)} & =0.05 b_{\lim } \text { with } \\
b_{\lim } & =\frac{1}{2 n_{\text {Dim }}} \frac{\Delta x^{2}}{\Delta t^{(n-1)}}\left[1-2 \max \left(\mathrm{Co}_{\phi}^{(n-1)}\right)\right] \\
n_{\text {Dim }} & =\left\{\begin{array}{l}
3(3 \mathrm{D}) \\
2(2 \mathrm{D})
\end{array},\right.
\end{aligned}
$$

where $\mathrm{Co}_{\phi}$ is the Courant number for the phase-field equation. This formulation of $b_{\text {lim }}$ comes from the discretization of the phase-field equation. The factor $1 /\left(2 n_{\text {Dim }}\right)$ stems for the use of the simplest stencils for the term $\nabla^{2} \phi$ (5-point stencil in 2D and 7-point stencil in 3D). The $b$ used here was somewhat smaller than the recommendation given by Sun and Beckermann [27], which was to use $b \approx 0.4 b_{\text {lim }}$, where $b_{\lim } \approx 1.2 \mathrm{Wa}$ was the observed stability limit and $a$ the characteristic normal front speed. We should note that this recommendation was obtained for interface motion with a uniform normal speed or a uniform translation speed. In our case, the problem is more complex as the envelope needs to deform due to nonuniform growth velocity. We observed that a too high stabilization parameter constrains the shape too much. Indeed, the $\phi$-profile has to expand slightly in some places to allow the envelope to bend. The influence of $b$ on the overall envelope shape is discussed below.

The grid spacing should be chosen with respect to the characteristic length scales of the problem. In addition to the diffusion length, the radius of curvature of the envelope appears as a length scale. The diffusion length is smallest at the tip of the primary arm and is $l_{\text {diff }}=D_{1} / V_{\mathrm{LGK}}$. The smallest curvature of the envelope $R_{\text {env,min }}$ also appears at the tip of the primary arm, but is more difficult to estimate a priori. We can assume that it scales with the tip radius in a 
Table 1

Physical and numerical parameters, and relations between different characteristic length scales, the grid spacing, $\Delta x$, and the size of the computational domain, $z_{\max }$, for (a) the grid convergence study and (b) the sensitivity study assessing the influence of the stabilization parameter $b$. Both studies are in 2D. A stagnant film thickness of $\delta / l_{\text {diff }}=1$ was used.

\begin{tabular}{|c|c|c|c|c|c|}
\hline$\Omega_{0}$ & $\frac{l_{\text {diff }}}{R_{\mathrm{LGK}}}$ & $\frac{z_{\max }}{l_{\text {diff }}}$ & $\frac{l_{\text {diff }}}{\Delta x}$ & $\frac{\Delta x}{R_{\mathrm{LGK}}}$ & $\frac{b}{b_{\lim }}$ \\
\hline \multirow{2}{*}{0.179} & \multirow{2}{*}{38.2} & \multirow{2}{*}{25} & 3 to 45 & 12.7 to 0.85 & 0.05 \\
\hline & & & 10 & 3.82 & 0.004 to 0.9 (b) \\
\hline
\end{tabular}

similar way as the whole envelope. Following this assumption, the ratio of the smallest curvature to the diffusion length scales as $R_{\text {env,min }} / l_{\text {diff }} \sim f P e_{\mathrm{LGK}}$. The proportionality factor $f$ is larger than 2 , since the envelope curvature radius is necessarily larger than the tip radius. This means that at very high supersaturations (when $P e_{\mathrm{LGK}} \gg 1$ ) the grid size will be controlled mainly by the diffusion length, whereas at very low supersaturations it will be controlled by the envelope curvature at the primary tip. A simple estimation shows that the threshold between these two regimes is well above a supersaturation of $\operatorname{Iv}(0.5)=0.46$. Such supersaturations are not of great interest since the experiments used for validation [18] were performed at considerably lower values. In technical processes the supersaturations are even much lower. This means that the pertinent regime to check the influence of the grid spacing is at low supersaturation, in order to clearly discern the limits presented by the envelope curvature.

Because the grid convergence study required a large number of computations on dense grids, it was performed in 2D. An initial supersaturation of $\Omega_{0}=0.179$ was used. The Péclet number of a $2 \mathrm{D}$ Ivantsov tip growing at this supersaturation is identical to the Péclet number of a 3D tip growing at a $\Omega_{0}=0.05$, which was the smallest supersaturation in the 3D computations that follow. In this case the primary dendrite tip radius is around 40 times smaller than the diffusion length at the tip. All computations were performed on a regular square mesh for $1 / 4$ of the $2 \mathrm{D}$ grain on a computational domain of length $z_{\max }=25 l_{\text {diff }}$, which was sufficiently large to obtain a steady state of the envelope tip. The physical and numerical parameters are detailed in Table 1.

The critical result of interest is the shape of the envelope of a primary arm. We compared the width of the envelope as a function of the distance from the envelope tip $\left(X_{\text {act }}(z)\right.$ according to the notations in Fig. 2) for 13 grids. An estimation of the continuum solution (i.e. at zero grid spacing) of the envelope shape was obtained by Richardson extrapolation. [32]. With this reference, the error of the envelope width, $X_{\text {act }}$, along the primary arm, shown in Fig. 6, was estimated. We can see that the relative errors are constant along a large part of the dendrite axis, sufficiently far from the tip. For a grid spacing of $\Delta x=0.10 l_{\text {diff }}=3.8 R_{\mathrm{LGK}}$, the error is about $5 \%$. The errors increase drastically only in the close vicinity of the tip. This means that the shape of the envelope can be predicted accurately even if the tip of the envelope is

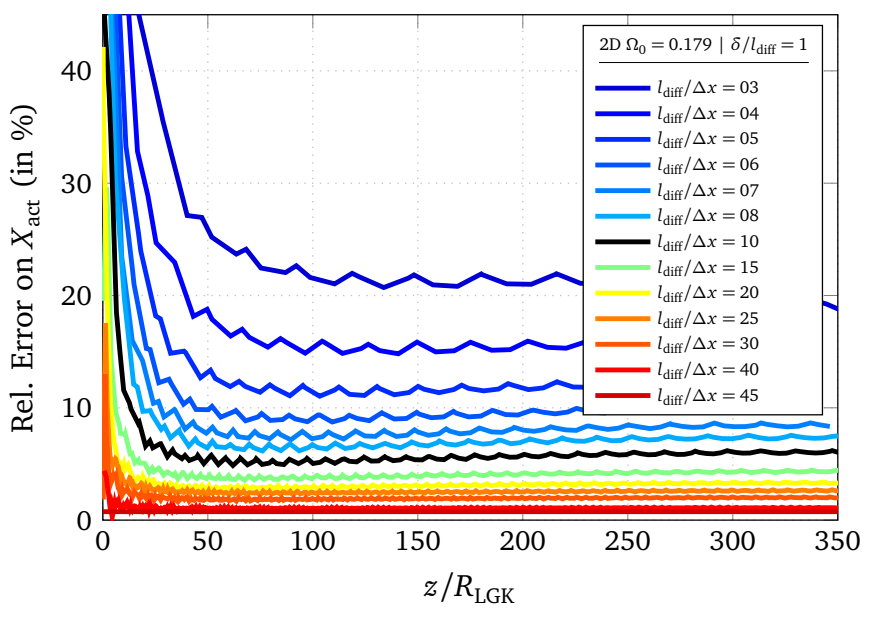

Fig. 6 Relative error of the envelope width along the primary dendrite arm for 13 grids. $z$ is the distance from the primary tip. The error is computed with respect to an estimation of the continuum solution (i.e. at zero grid spacing) of the envelope shape obtained by Richardson extrapolation.

not finely resolved. An accurate resolution of the envelope shape at the tip is thus not of prime importance. Moreover, the envelope does not have any physical meaning close to the tip, before the onset of branching. The convergence of the envelope width is shown in more detail in Fig. 7 in the form of the error in six probe points at different distances from the primary tip. The observed order of convergence is approximately 1 . The convergence seems to improve with very fine grids, where the error is however already within a few percent.

An adaptive time step was used. In all computations it was set such that both the Courant number for the phasefield equation, $\mathrm{Co}_{\phi}$, and the grid Fourier number for the solute equation, $\mathrm{Fo}_{\Delta x}=D_{1} \Delta t / \Delta x^{2}$, remained smaller than 0.1. This ensured a sufficient accuracy of the time integration.

The stabilization parameter, $b$, also has an influence on the predicted envelope shapes. We quantified the influence by a parameter study, varying the ratio $b / b_{\text {lim }}$ from 0.004 to 0.9 , as given in Table 1 . Fig. 8 shows the dependence of the envelope width, $X_{\text {act }}$, on $b / b_{\text {lim }}$ in six probe points at different distances from the primary tip. We can see that there is no clear convergence point. The variations over the range $0.05<b / b_{\lim }<0.4$ are around $2.5 \%$. This is much less than the errors related to the spatial discretization. The dependence of the choice of $b / b_{\lim }$ on the supersaturation is not explicitly analyzed here. However we can understand it through the analyses of Sun and Beckermann [27]. They have shown that the error linked to the stabilization of the diffuse interface in this interface tracking method depends on the dimensionless stabilization parameter $b^{\prime}=b /\left(W V_{\mathrm{LGK}}\right)$. Accounting for our previously justified choices of the numerical parameters ( $W$ is proportional to $R_{\mathrm{LGK}}$ and $b_{\mathrm{lim}}$ is proportional to $\Delta x^{2} / \Delta t$ in the limit of small Courant numbers), we can deduce that $b \sim\left(b / b_{\lim }\right) \mathrm{Pe}_{\mathrm{LGK}}^{-1} \mathrm{Fo}_{\Delta x}^{-1}$. This shows that the Péclet number should affect the error in the same way as $\left(b / b_{\text {lim }}\right)^{-1}$. In our 3D computations $P e_{\mathrm{LGK}}$ varies from 0.013 


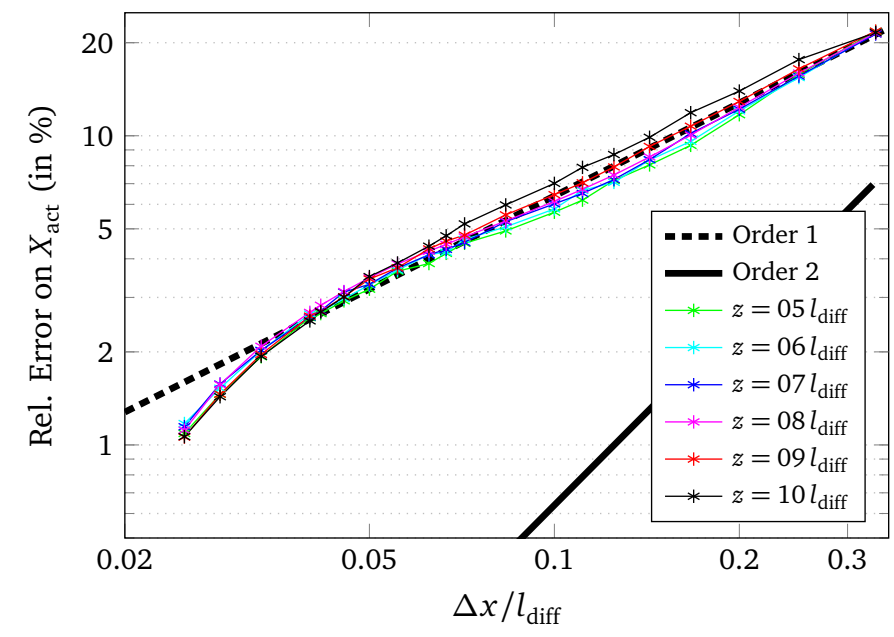

Fig. 7 Grid convergence of the envelope width in six probe points along the primary dendrite arm. $z$ is the distance from the primary tip. The error is computed with respect to an estimation of the continuum solution (i.e. at zero grid spacing) of the envelope shape obtained by Richardson extrapolation. The dashed line shows a fit of a first-order discretization error.

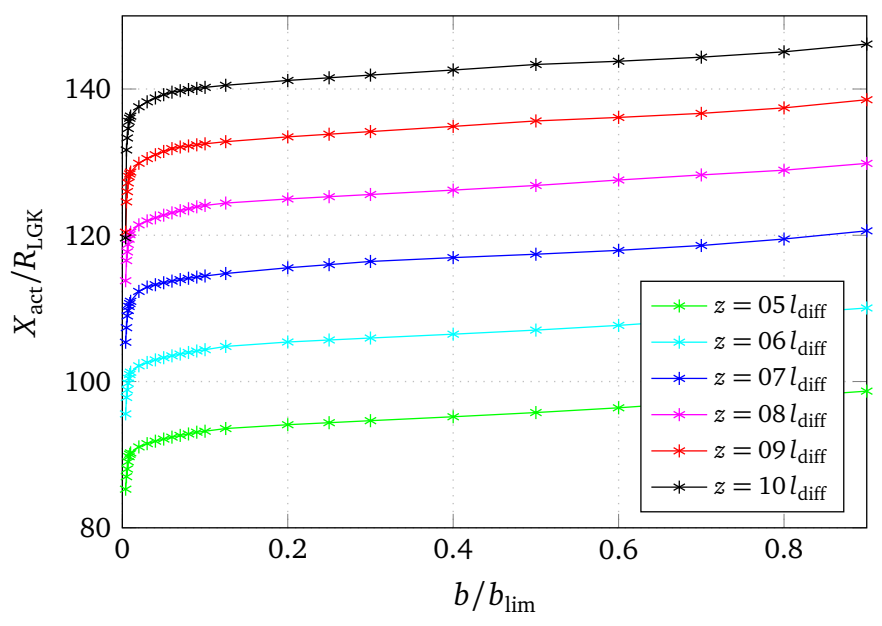

Fig. 8 The dependence of the envelope width on the stabilization parameter $b$ in six probe points along the primary dendrite arm. $z$ is the distance from the primary tip.

to 0.062 (i.e. by a factor of 4.8 ) as $\Omega_{\infty}$ varies from 0.05 to 0.25 . This should affect the solution in the same way as a reduction of $b / b_{\text {lim }}$ by a factor of around 0.2 . Fig. 8 indicates that this changes the envelope width by only around $2 \%$.

\section{Free growth of an equiaxed grain}

Steinbach et al. $[14,15]$ demonstrated the validity of the mesoscopic model for a pure substance. In this paper, we study the validity of the model for a binary alloy. We are primarily interested in all factors that will affect the fidelity of the representation of interactions between several neighboring grains - the raison d'être of this model. The principal factors are the growth velocity, the shape of the grain envelopes, and the quantity of solute rejected into the liquid surrounding the envelope. The simplest configuration to analyze this is the growth of an isolated equiaxed grain into an infinite and uniformly supercooled melt. While this is an idealized configuration, reliable quantitative references are available, allowing for a rigorous validation.

The predictions of the primary tip speed can be validated against the Ivantsov solution combined with the tip selection criterion. However, simple solutions do not exist for the envelope shape or the solute flux from a dendrite. Validation is therefore made by comparisons with the recent experiments of Melendez and Beckermann [18]. They performed detailed characterizations of equiaxed dendrites of transparent succinonitrile-acetone alloys growing into a uniformly undercooled melt. They deduced generalized scaling laws for the dendrite envelope shape and for the solid fraction inside the envelope, which is a direct indicator of the quantity of the rejected solute. Although these alloy dendrites were governed by both heat and solute transport (with different proportions of thermal and solutal undercooling) the obtained scaling correlations are close to those observed for pure materials in microgravity. It is therefore reasonable to assume that the same generalized envelope shape also holds for purely solutal dendrites with negligible thermal undercooling. Our comparisons are based on this premise and our validation is done for isothermal, purely solutally driven growth.

\subsection{Validation cases}

We consider a tridimensional isolated equiaxed grain growing by solute diffusion into an infinite undercooled melt. The only physical parameter in this case is the supersaturation of the melt, $\Omega_{0}$. The computations were performed for supersaturations in the range of $\Omega_{0}=0.05-0.25$. This is similar to the range of solutal supersaturations of $\Omega_{0} \approx 0.05-0.45$ found in the experiments [18]. These were estimated with the LGK model from the known total undercoolings. Initially, the entire domain is liquid at a uniform supersaturation $\Omega_{0}$. A spherical nucleus is introduced, an envelope within which the liquid has zero supersaturation and the solid fraction is given by the Scheil law. At the domain boundaries, conditions of zero normal first derivative were used for the fields $\phi$ and $\Omega_{1}$.

There are no expected limitations to using the model at a lower or higher supersaturation as long as the grain morphology is dendritic. The explored range of supersaturation is merely a question of achievable/convenient domain size because we wanted to compare our results to free-growth experiments. Indeed, the size of the domain must be sufficient to ensure that the diffusion field around the grain is not affected by the confinement before the envelope of the primary arm reaches a steady state, i.e. when both the primary tip speed and the envelope shape no longer vary. Generally, the steady state was reached at $\tau=t V_{\mathrm{LGK}} / l_{\mathrm{diff}} \approx 5$, which corresponds roughly to a dendrite arm length of $5 l_{\text {diff. The }}$ domain sizes were between $10 l_{\text {diff }}$ and $29 l_{\text {diff }}$, as specified in Table 2, which was sufficiently large.

All the computations presented in this section were performed on a uniform structured hexahedral mesh with $200 \times$ $200 \times 200$ volumes for $1 / 8$ of the grain. For each case the 
Table 2

Physical and numerical parameters used in the 3D computations investigating the dependence of the predictions of free growth of an equiaxed grain on the stagnant film thickness. The grid spacing, $\Delta x$, and domain size, $z_{\max }$, (corresponding to the maximal possible length of the primary dendrite arm) are compared to the characteristic scales of the problem.

\begin{tabular}{ccccccc}
\hline$\Omega_{0}$ & $\frac{l_{\text {diff }}}{R_{\mathrm{LGK}}}$ & $\frac{\Delta x}{R_{\mathrm{LGK}}}$ & $\frac{\Delta x}{l_{\text {diff }}}$ & $\frac{z_{\max }}{R_{\mathrm{LGK}}}$ & $\frac{z_{\max }}{l_{\text {diff }}}$ & $\frac{\delta}{l_{\text {diff }}}$ tested \\
\hline 0.05 & 38.2 & 3.0 & 0.08 & 600 & 15.7 & $0.16-2.62$ (20 values) \\
\hline 0.10 & 14.7 & 2.0 & 0.14 & 400 & 27.3 & $0.27-2.73$ (12 values) \\
0.15 & 8.01 & 1.0 & 0.12 & 200 & 25.0 & $0.37-2.25$ (10 values) \\
0.20 & 5.06 & 0.5 & 0.10 & 100 & 19.8 & $0.30-2.77$ (10 values) \\
0.25 & 3.46 & 0.5 & 0.14 & 100 & 28.9 & $0.43-3.47$ ( 9 values) \\
\hline
\end{tabular}

grid size $\Delta x$ was chosen in accordance with the theoretical diffusion length $l_{\text {diff }}=D_{1} / V_{\mathrm{LGK}}$ at the primary tip. Typically, $\Delta x \approx 0.1 l_{\text {diff }}$ allows for accurate resolution of the solute diffusion field. The domain size was $z_{\max } \sim 20 l_{\text {diff }}$, which was sufficient in all cases, as shown above. All parameters are detailed in Table 2 .

\subsection{Primary tip speed}

The dependence of the primary tip speed on the supersaturation as given by the mesoscopic model is shown in Fig. 9. The primary tip speed, $V_{\text {tip }}$, that was determined by tracking the position of the primary tip with time, is compared to the theoretical speed, $V_{\mathrm{LGK}}$, which is considered to be the reference speed that the model should ideally predict. Several different values of the stagnant film thickness, $\delta$, were used in the mesoscopic computations and the plot reveals a certain dependence of the predictions on this model parameter. A clearer presentation of the influence of the stagnant film thickness is given by Fig. 10. The theoretical diffusion length, $l_{\text {diff }}=D_{1} / V_{\mathrm{LGK}}$, is used to scale the stagnant film thickness in order to try to reveal a general relationship. Overall, we can see that the errors in the predicted primary tip speed are large for small stagnant film thicknesses. However, whatever the supersaturation, the predicted primary tip speed approaches the theoretical speed when the stagnant film thickness is of the same magnitude as the diffusion length $D_{1} / V_{\mathrm{LGK}}$. Within a large range of supersaturations the error is less than $20 \%$ when $\delta / l_{\text {diff }}>1$ and less than $10 \%$ when $\delta / l_{\text {diff }}>1.5$.

This behavior can be explained by the matching of the analytical solution for the dendrite tip, Eq. (1), to the mesoscopic solution of the diffusion field around the envelope. This is illustrated in Figs. 11a and 11b, which show the matching of the concentration fields along the axis of a primary arm in the vicinity of the tip for two different supersaturations, $\Omega_{0}=0.10$ and $\Omega_{0}=0.25$, respectively. In each figure the mesoscopic solutions of the concentration field around the envelope for two different values of the dimensionless stagnant film thickness $\delta / l_{\text {diff }}$ and the analytical solution of the concentration field around the tip (LGK model) are plotted. The mesoscopic field and the tip field are inherently different because the envelope is distinct from the liquid-solid in-

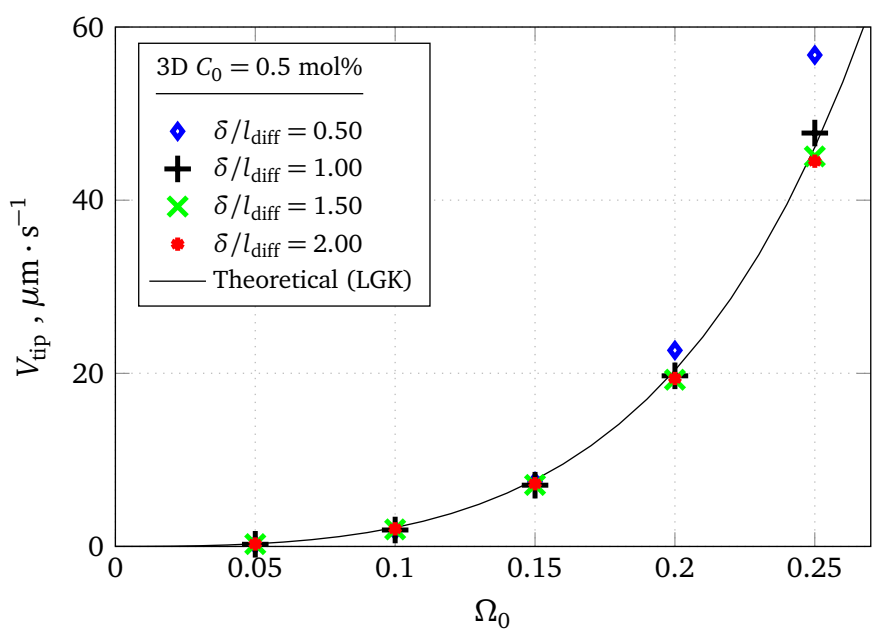

Fig. 9 Predicted primary tip speed versus the supersaturation for different values of the stagnant film thickness normalized by the diffusion length. The dimensional values are given for succinonitrile- $0.5 \mathrm{~mol} \%$ acetone (with $\sigma^{*}=0.02$ and thermophysical properties from $\left.[18,33,34]\right)$.

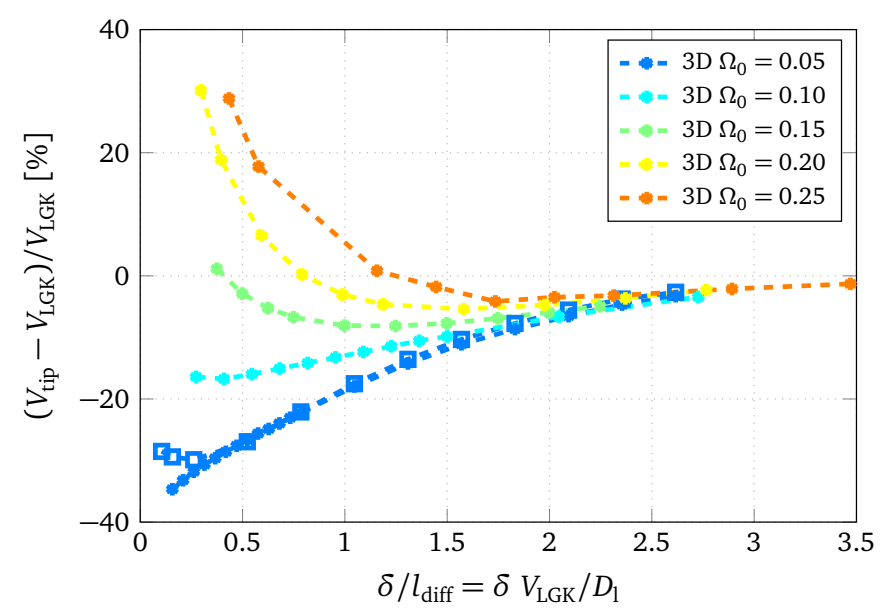

Fig. 10 Dependence of the relative error of the predicted primary tip speed on the stagnant film thickness normalized by the diffusion length.

terface. While the envelope is at the same supersaturation and advances at the same speed as the tip, it has a different shape and a larger radius of curvature at the end of the primary arm. As can be seen in Figs. 11a and 11b, the matching of the two solutions at a distance smaller than $l_{\text {diff }}$ can lead to errors. The supersaturation at the confocal envelope and hence the tip speed can be either underestimated (generally at low supersaturations, such as $\Omega_{0}=0.10$ in Fig. 11a) or overestimated (at high supersaturations and small stagnant film thicknesses, such as $\Omega_{0}=0.25$ and $\delta / l_{\text {diff }}=0.43$ in Fig. 11b). On the opposite, if the matching is done at a sufficiently large distance, corresponding to several diffusion lengths $l_{\text {diff }}$, the supersaturation at the confocal envelope approaches the far-field nominal supersaturation of the system, $\Omega_{0}$, independently of all model parameters. The primary tip speed, $V_{\text {tip }}$, then approaches the theoretical speed, $V_{\mathrm{LGK}}$. The reason is that for $\delta \gg l_{\text {diff }}$ the stagnant-film formulation approaches the original far-field formulation of the Ivantsov so- 

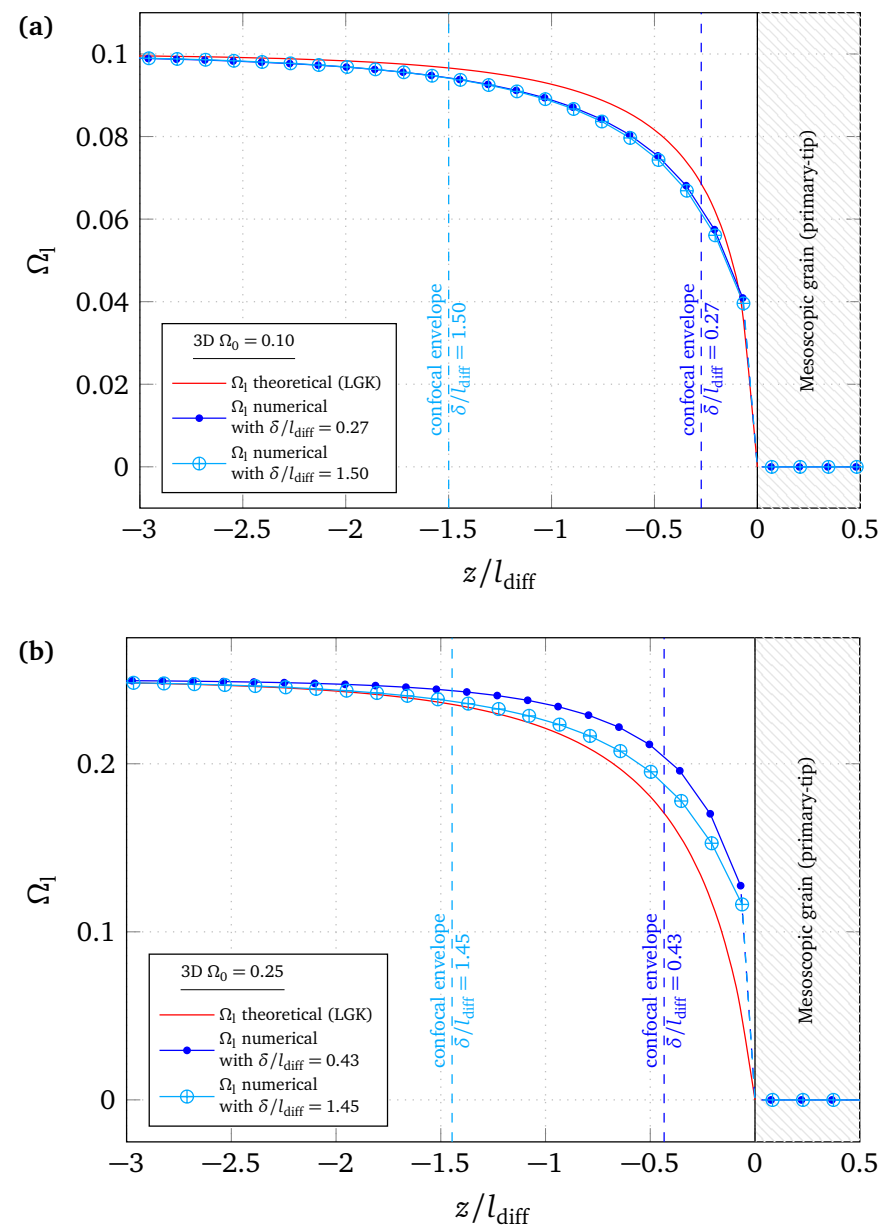

Fig. 11 Comparison of the mesoscopic (numerical) and the microscopic (theoretical) profiles of supersaturation in the liquid ahead of the primary dendrite tip. The microscopic tip profile is computed for a tip operating at the theoretical speed $V_{\mathrm{LGK}}$. In each case mesoscopic profiles for a small and for a moderate value of the normalized stagnant film thickness $\delta / l_{\text {diff }}$ are shown. (a) Case of a low supersaturation $\left(\Omega_{0}=0.10\right)$, where the tip speed is underestimated at small $\delta / l_{\text {diff. }}$. (b) Case of a high supersaturation $\left(\Omega_{0}=0.25\right)$, where the tip speed is overestimated at small $\delta / l_{\text {diff. }}$.

lution. This might lead one to believe that a large stagnant film thickness should be used in order to ensure good accuracy of the model. However, as we will show in the next section, too large values of $\delta / l_{\text {diff }}$ lead to erroneous predictions of the envelope shape and should be avoided. Sufficiently accurate matching is ensured at all supersaturations if $\delta / l_{\text {diff }}>1$, as shown in Figs. 10 and 11 .

\subsection{Envelope shape}

The mesoscopic model is further validated by comparing predicted envelope shapes to measurements from experiments involving the growth of a dendrite into a uniformly supersaturated binary melt. Melendez and Beckermann [18] showed that the sidebranch structure of alloy dendrites is such that the length of the active sidebranches, $X_{\text {act }}$, follows a self-similar power law with distance from the tip, $z$ :

$$
\begin{gathered}
\frac{X_{\text {act }}}{R_{\text {tip }}}=A_{1}\left(\frac{z}{R_{\text {tip }}}\right)^{n_{1}} \\
A_{1}=0.84 \quad n_{1}=0.85
\end{gathered}
$$

This law is universal and is independent of the undercooling and of the alloy concentration. It showed an excellent fit to the experimental data for $10<z / R_{\text {tip }}<200$, which corresponds roughly to the range of the so-called self-similar sidebranching regime $10<z / R_{\text {tip }}<1 / \mathrm{Pe}_{\text {tip }}$. Note that sidebranching starts behind the tip at a distance of about $z / R_{\text {tip }}=10$ for pure dendrites and at about $z / R_{\text {tip }}=6$ in SCNacetone alloys [18] and therefore the envelope does not have any physical meaning closer to the tip of the primary arm. The experiments leading to Eq. (12) were performed under terrestrial gravity, therefore some influence of natural convection is inevitable. By comparing Eq. (12) to scaling laws for pure dendrites under terrestrial gravity [18] and microgravity [19], Melendez and Beckermann have shown however, that the influence of convection on the envelope width is rather small for branches that are approximately downward growing. The difference of the envelope width compared to pure dendrites in microgravity is around $20 \%$. This difference is not due to convection, but due to the earlier onset of sidebranching in alloys. The influence due to convection can be estimated by comparing scaling laws for pure dendrites in micro- and terrestrial gravity [18], which shows that the convection tends to widen the envelope at distances from the tip of about $z / R_{\text {tip }}>100$.

The results of the mesoscopic model are presented in Figs. 12 and 13. As we can see in Fig. 12, the stagnant film thickness used in the computations affects the shape of the grain and the distribution of the solid phase in the interior of the envelope. Steady-state envelope contours for three different supersaturations in the range $\Omega_{0}=0.05-0.25$ are shown in Fig. 13. For each supersaturation the computations were performed using several different stagnant film thicknesses, indicated in the figures in the dimensionless form $\delta / l_{\text {diff. The }}$ normalized envelope width, $X_{\text {act }} / R_{\text {tip }}$, follows a power law with the normalized distance from the tip, $z / R_{\text {tip }}$, in all cases. Fig. 13 shows the absolute difference between the predicted envelope width and the scaling law. Generally, the envelope width increases with a larger stagnant film thickness. This can be explained by the matching between the analytical tip solution and the mesoscopic concentration field around the envelope. The larger the distance of the matching, the higher is the local (confocal) supersaturation that the secondary tips feel and the faster is the lateral growth of the envelope. When the stagnant film thickness becomes much larger than the diffusion length $\left(\delta / l_{\text {diff }} \gg 1\right)$, the supersaturation at the confocal envelope becomes identical along the whole envelope, and equal to $\Omega_{0}$. The tip speeds are then also identical along the whole envelope and they approach $V_{\mathrm{LGK}}$. The grain then takes an octahedral shape as can be seen in Fig. $13 \mathrm{c}$ for $\delta / l_{\text {diff }}=3.47$. Conversely, for sufficiently small stagnant film thicknesses $\left(\delta / l_{\text {diff }} \lesssim 1\right)$ the variation of 


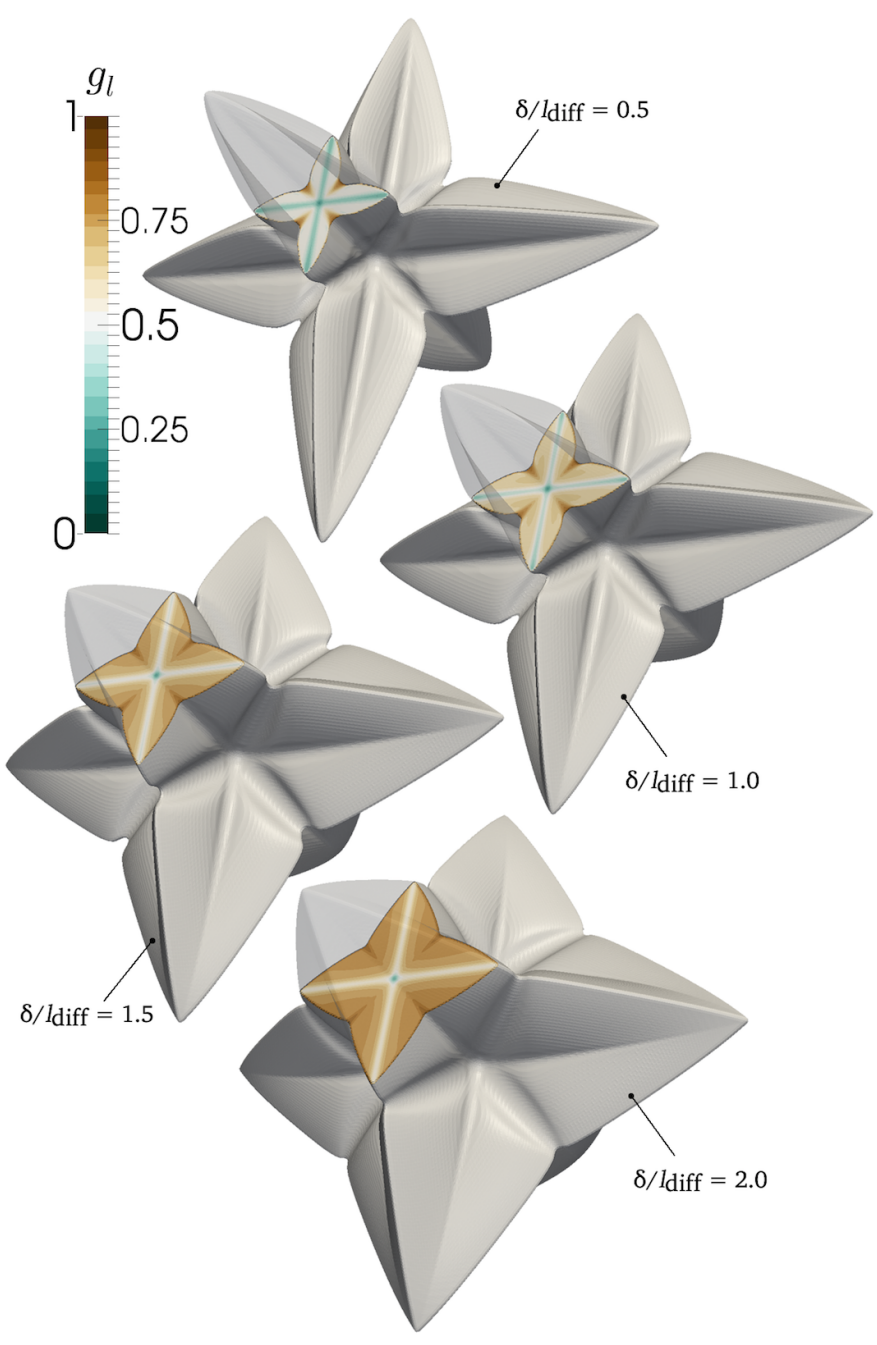

Fig. 12 Comparison of equiaxed grains obtained for an initial supersaturation $\Omega_{0}=0.15$ and different values of the normalized stagnant film thickness, $\delta / l_{\text {diff }}$.

the local supersaturation felt by the tips along the envelope is larger and the associated tip speeds vary enough to give the grains their characteristic shape. In Fig. 13 we can see that the width of the envelope is closest to the experimental scaling law when using a stagnant film thickness of about $\delta / l_{\text {diff }} \approx 0.5$. This holds for all supersaturations, except for the lowest supersaturation $\left(\Omega_{0}=0.05\right)$, where a somewhat smaller value of $\delta / l_{\text {diff }}$ gives a better fit.

However, as we have shown, a correct estimation of the primary tip speed requires larger values, of about $\delta / l_{\text {diff }}>1$; at smaller values the errors become significant. A reasonable compromise to reconcile these opposing requirements is to use a stagnant film thickness of around $\delta / l_{\text {diff }} \sim 1$. In this way both the primary tip speed and the envelope width can be estimated within about $20 \%$ accuracy.

\subsection{Projected solid area}

In their experiments Melendez and Beckermann [18] also measured the solid projection area, $F$, of the SCN-acetone
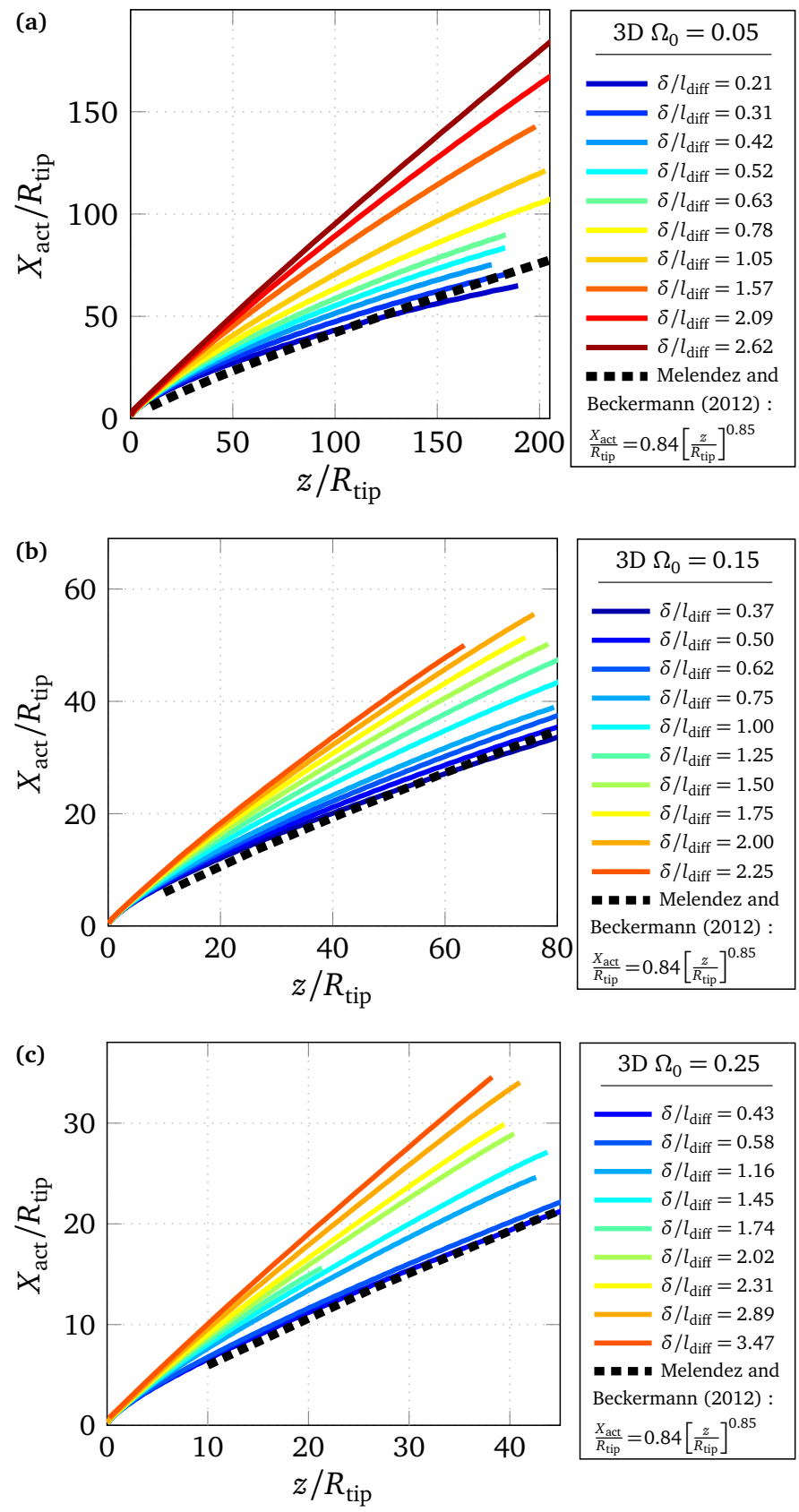

Fig. 13 Normalized envelope widths, $X_{\text {act }} / R_{\text {tip }}$, as a function of the dimensionless longitudinal distance from the tip, $z / R_{\text {tip }}$, for initial supersaturations of: (a) $\Omega_{0}=0.05$, (b) $\Omega_{0}=0.15$, and (c) $\Omega_{0}=0.25$.

dendrites, as defined in Fig. 2. They observed that the projection area as a function of the distance from the tip, $z$, follows a universal self-similar scaling law given by:

$$
\begin{gathered}
\frac{F}{R_{\text {tip }}^{2}}=A_{2}\left(\frac{z}{R_{\text {tip }}}\right)^{n_{2}} \\
A_{2}=0.58 \quad n_{2}=1.77
\end{gathered}
$$

These values of the exponent $n_{2}$ and of the amplification factor $A_{2}$ are very close to the values found for pure substances in terrestrial gravity [18] and microgravity [19]. The same scaling law thus appears to be valid for all solute con- 
centrations (including pure substances), undercoolings, and even convection intensities if we consider only the downward growing branches as in the experiments [18].

In the mesoscopic model the projection area can be related to the solid fraction field in the envelope. We calculated an equivalent of the projection area by first extracting the plane of the ridge of the sidebranches, (i.e. the plane at $y=0$ ) and then calculating the area integral of the solid fraction as a function of the distance from the primary tip in this plane. With the notations of the Fig. 2, the projected area is calculated using the formula:

$$
F(z)=\int_{0}^{z} \int_{0}^{X_{\text {act }}(z)} g_{\mathrm{s}}(x, z) \mathrm{d} x \mathrm{~d} z
$$

In Fig. 14, we can see that the projection area is in very good agreement with the experiments for all supersaturations. Moreover, we can conclude that the stagnant film thickness has very little influence on the projection area.

Finally we can recommend $\delta / l_{\text {diff }}=1$ as a good compromise to obtain realistic simulations with the mesoscopic envelope field model in terms of envelope shape, internal solid fraction and primary tip speed.

\section{Solutal interaction between multiple equiaxed grains}

To demonstrate the power of the mesoscopic envelope model, we present several simulations involving a large ensemble of interacting equiaxed grains. Figure 15 shows simulations of randomly distributed and oriented grains growing isothermally in a cubic enclosure at an initial supersaturation of $\Omega_{0}=0.05$ and at three different grain densities. The dimensionless grain density, $N_{V}^{\text {ldiff }}$, representing the mean number of grains in a volume of $l_{\text {diff }}^{3}=\left(D_{1} / V_{\mathrm{LGK}}\right)^{3}$, varies from $1.25 \cdot 10^{-4}$ to $4 \cdot 10^{-3}$. This means that the mean distance between the grain centers $\bar{d}_{\mathrm{cc}}$ varies from $22 l_{\text {diff }}$ to $7 l_{\text {diff }}$. This distance is estimated based on a close regular packing arrangement (FCC or HCP), which gives $\bar{d}_{\mathrm{cc}}=$ $l_{\text {diff }}\left(N_{V}^{\text {ldiff }} / \sqrt{2}\right)^{-1 / 3}$. At the lowest grain density, shown in Fig. 15a, the grain interactions are very weak. Fully developed envelope shapes, similar to that of a free grain, develop well before the solutal interactions start. At the intermediate grain density, shown in Fig. 15b, we can see a pronounced interaction before the grains can reach a free-growth shape. The envelopes adapt to the neighbors. Only some of the primary branches develop a shape close to that of a freely growing primary arm envelope. At the highest grain density, shown in Fig. 15c, interactions occur very soon and the grains develop virtually octahedral shapes. These computations were done with a multi-phase-field formulation of the interface capturing algorithm, where each orientation is described by a distinct indicator field. Eq. (5) is then solved separately for each indicator field. This increases the computation time but is a very simple and robust way to account for multiple orientations. The parameters used in these computations are summarized in Table 3.
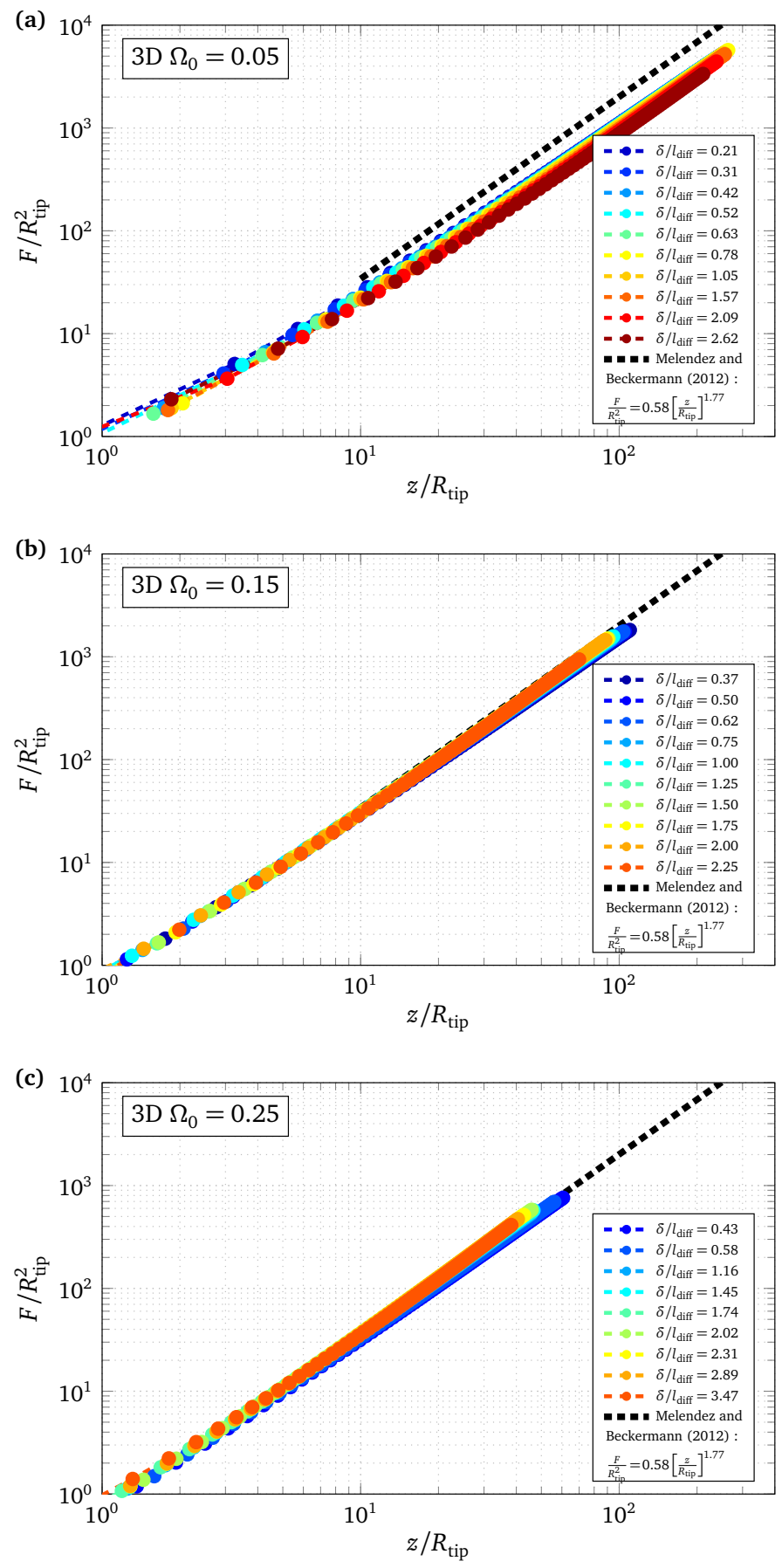

Fig. 14 Normalized projection area, $F / R_{\text {tip }}^{2}$, as a function of the dimensionless longitudinal distance from the tip, $z / R_{\text {tip }}$, for initial supersaturations of: (a) $\Omega_{0}=0.05$, (b) $\Omega_{0}=0.15$, and (c) $\Omega_{0}=0.25$.

Such simulations can provide important information and parameters for macroscopic volume-averaged models of solidification. We can think of the simulation domain as a representative elementary volume (REV) for the averaging. In this type of models [11], the equiaxed dendrite envelopes are assumed to have a simple shape (spherical, for example). The growth of an average envelope of this shape is then given by the velocity of the primary tips, which is calculated from the average supersaturation of the liquid outside the en- 
(a) 27 grains with 9 orientations in a cubic enclosure of volume $\left(60 l_{\text {diff }}\right)^{3}: N_{V}^{\text {ldiff }}=1.25 \cdot 10^{-4}\left(\bar{d}_{\mathrm{cc}}=22 l_{\text {diff }}\right)$.
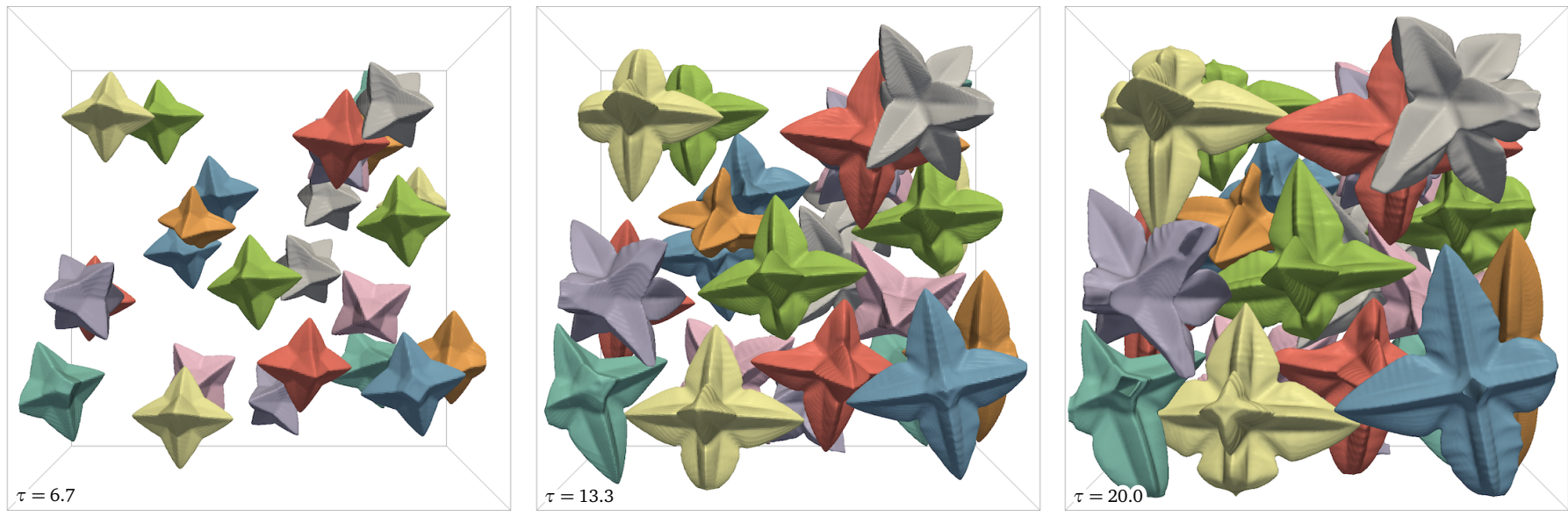

(b) 27 grains with 9 orientations in a cubic enclosure of volume $\left(30 l_{\text {diff }}\right)^{3}: N_{V}^{\text {ldiff }}=10^{-3}\left(\bar{d}_{\mathrm{cc}}=11 l_{\text {diff }}\right)$.
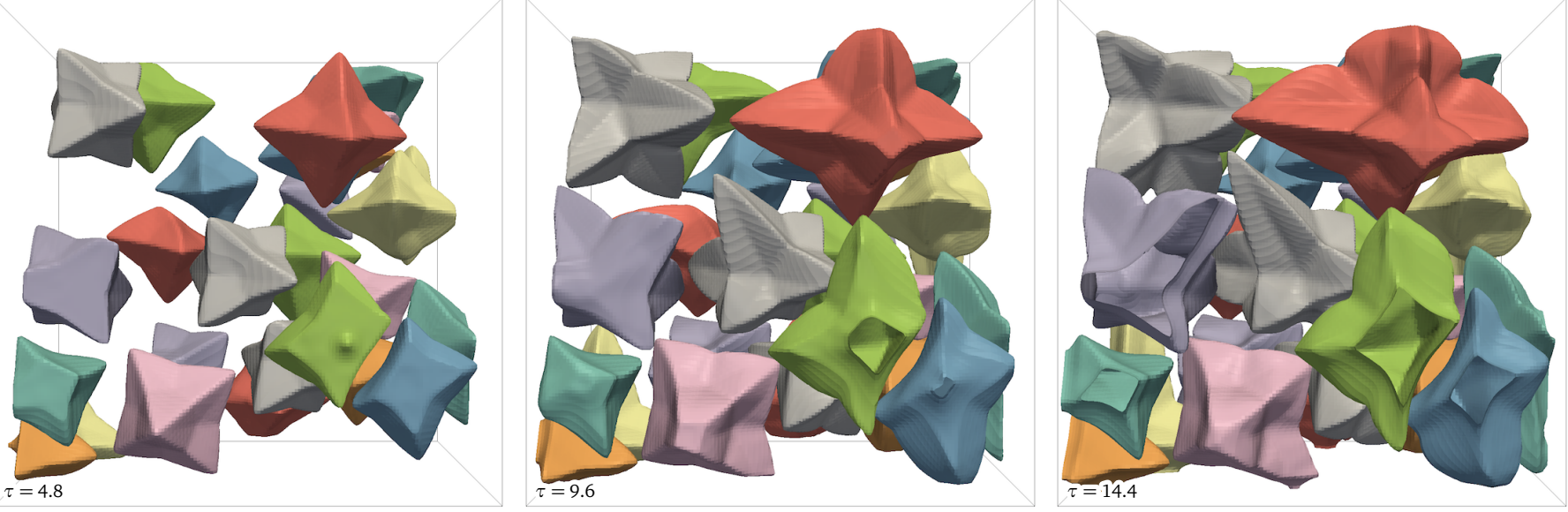

(c) 108 grains with 18 orientations in a cubic enclosure of volume $\left(30 l_{\text {diff }}\right)^{3}: N_{V}^{\text {ldiff }}=4 \cdot 10^{-3}\left(\bar{d}_{\mathrm{cc}}=7 l_{\text {diff }}\right)$.
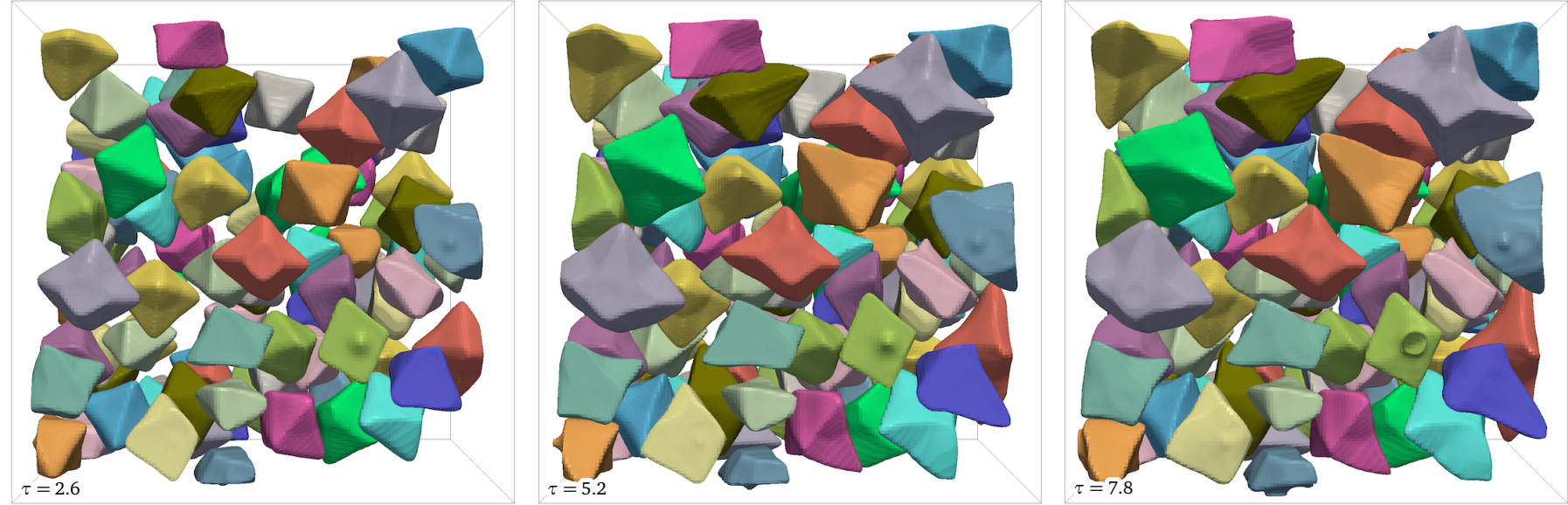

Fig. 15 Snapshots from simulations of randomly distributed and oriented grains growing isothermally in a cubic enclosure at an initial supersaturation of $\Omega_{0}=0.05$ and at three different dimensionless grain densities, $N_{V}^{\text {ldiff }}$ (or three different mean grain distances, $\bar{d}_{\mathrm{cc}}$ ). A stagnant film thickness of $\delta / l_{\text {diff }}=1.0$ was used in all simulations. 
Table 3

Physical and numerical parameters used in the 3D simulations of interactions between multiple grains. $n_{\text {grains }}$ and $n_{\text {orien }}$ are the number of grains and of different orientations, respectively. $L_{\mathrm{box}}$ is the length of the side of the cubical enclosure. The stagnant film thickness was $\delta / l_{\text {diff }}=1$ in all computations. The grid size, timestep and stabilization parameter were set such that: $\Delta x / l_{\text {diff }}=0.2,\left(\Delta t D_{1}\right) / \Delta x^{2}=0.25$, and $b / b_{\text {lim }}=0.05$. The approximate execution time is given for simulating the time $\tau=t V_{\mathrm{LGK}} / l_{\mathrm{diff}}=10$ on a 100 GFlops personal computer. The single grain simulation was done for $1 / 8$ of the grain by taking into account the symmetries.

\begin{tabular}{rrrrrrr}
\hline$n_{\text {grains }}$ & $n_{\text {orien }}$ & $\frac{L_{\text {box }}}{l_{\text {diff }}}$ & $N_{V}^{\text {ldiff }}$ & $\frac{\bar{d}_{\mathrm{cc}}}{l_{\text {diff }}}$ & $\frac{L_{\text {box }}}{\Delta x}$ & $\begin{array}{c}\text { exec. time } \\
\text { for } \tau=10\end{array}$ \\
\hline 1 & 1 & 40 & $1.56 \cdot 10^{-5} \approx 0$ & $45 \approx \infty$ & 200 & $<1$ hour \\
27 & 9 & 60 & $1.25 \cdot 10^{-4}$ & 22 & 300 & 7.0 days \\
27 & 9 & 30 & $10^{-3}$ & 11 & 150 & 2.6 days \\
108 & 18 & 30 & $4 \cdot 10^{-3}$ & 7 & 150 & 4.0 days \\
\hline
\end{tabular}

velopes (extradendritic liquid), using an Ivantsov-based dendrite growth model. The evolution of the average solute concentration of the extradendritic liquid in the isothermal REV, $\left\langle C_{l}\right\rangle^{\mathrm{e}}$, is described by an averaged conservation equation of the form

$$
\frac{\partial\left(g_{\mathrm{e}}\left\langle C_{\mathrm{l}}\right\rangle^{\mathrm{e}}\right)}{\partial t}=C_{1}^{*} \frac{\partial g_{\mathrm{e}}}{\partial t}+D_{1} S_{\mathrm{V}, \mathrm{env}} \frac{C_{1}^{*}-\left\langle C_{\mathrm{l}}\right\rangle^{\mathrm{e}}}{\delta_{\mathrm{l}}},
$$

where $g_{\mathrm{e}}$ is the volume fraction of the extradendritic liquid in the REV. The first term on the RHS of Eq. (15) gives the contribution due to the growth of the envelopes, which engulf liquid at the equilibrium concentration, $C_{1}^{*}$ (the concentration of the liquid in the envelopes). The second term on the RHS is the diffusion flux from the envelopes into the extradendritic liquid. It is formulated in terms of the average concentration gradient at the surface of the envelopes, which is approximated by the concentration difference between the envelope and the averaged extradendritic liquid, $\left(C_{1}^{*}-\left\langle C_{l}\right\rangle^{\mathrm{e}}\right)$, divided by a diffusion length, $\delta_{1}$. To give the flux, the gradient is multiplied by the diffusion coefficient, $D_{1}$, and by $S_{\mathrm{V}, \text { env }}$, the specific surface area of the envelopes per unit volume of the REV. An accurate formulation of the diffusion length, $\delta_{1}$, and of the specific surface area, $S_{\mathrm{V}, \text { env }}$, is essential for an accurate representation of the envelope growth kinetics. Both are usually modeled by simplistic analytical or empirical relations. Now the averaging can be done from the mesoscopic simulations, which represent the phenomena at the scale of the macroscopic REV in detail. The evolution of the envelope surface area in the simulations of interacting grains was determined directly from the computed envelope shapes. The diffusion length was determined indirectly by solving (15) for $\delta_{1}$, which requires first to determine $\left\langle C_{l}\right\rangle^{\mathrm{e}}$ and $g_{\mathrm{e}}$ from the mesoscopic simulations.

Figure 16 shows the evolution of the specific envelope surface area in terms of the average envelope sphericity. The sphericity, $\Psi$, is the ratio of the surface area of a sphere with the same volume as the envelope to the surface area of the envelope. For a spherical envelope, $\Psi=1$, and for any other shape, $\Psi<1$. For a fixed number of grains the sphericity and the specific envelope surface area are linked by the pro-

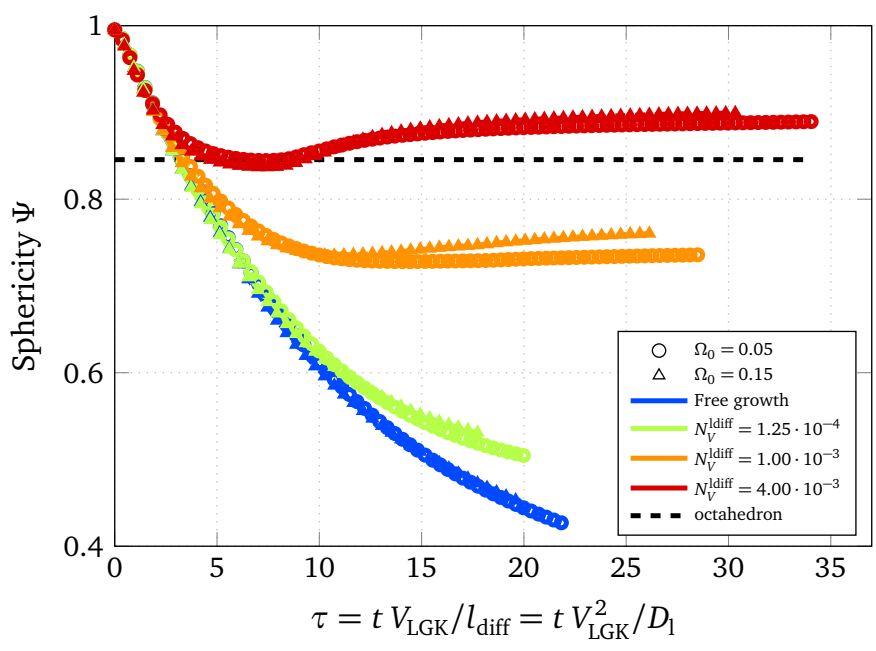

Fig. 16 Evolution of the average sphericity of the envelope over the dimensionless time.

portionality $S_{\mathrm{V} \text {,env }} \propto g_{\mathrm{e}}^{2 / 3} \Psi$. In addition to the cases shown in Fig. 15, a case of free growth of an isolated grain, and a set of cases for a higher supersaturation $\left(\Omega_{0}=0.15\right)$ and the same grain densities are included. The time in the plots is scaled by the diffusion time at the scale of the diffusion length, $l_{\mathrm{diff}}=D_{1} / V_{\mathrm{LGK}}$, which can also be interpreted as the traveling time of a primary tip growing at the free-growth speed $V_{\mathrm{LGK}}$ across the diffusion length $l_{\text {diff }}$.

It is interesting to compare the average sphericity of the computed envelopes to approximations of the envelope shape made in different macroscopic volume-averaged models. In early models spherical envelopes with a radius equal to the primary arm length were often used [11, 35, 36]. The sphericity is then equal to 1 . Other models $[37,38]$ use octahedral envelopes with a sphericity of $(\pi /(3 \sqrt{3}))^{1 / 3} \approx 0.846$. or shapes composed of six orthogonal square pyramids [12], with a sphericity of $(484 \pi) /(12+\sqrt{(3)} \approx 0.837$, i.e. very close to that of an octahedron.

We can see that starting from the initially spherical nuclei the sphericity first decreases in a manner that is independent of the supersaturation and of the grain density. In the case of free growth the sphericity continuously decreases. In the two cases with the highest grain density the sphericity first passes through a minimum and through a stage of spheroidization and then tends to a limit value. Both, the limit value and the entire evolution in terms of the dimensionless time, depend on the grain density, but not on the supersaturation. The departure from the free-growth behavior occurs when the diffusion layers of adjacent grains start to interact. This point of departure can be estimated by the dimensionless time of $\tau_{\mathrm{d}} \sim\left(D_{1} / l_{\text {diff }}^{2}\right)\left(\bar{d}_{\mathrm{cc}} / 2-l_{\text {diff }}\right) / V_{\mathrm{LGK}}=\bar{d}_{\mathrm{cc}} /\left(2 l_{\text {diff }}\right)-1$. For the three grain densities, from the highest to the smallest, this relation gives $\tau_{\mathrm{d}} \sim 2.5, \tau_{\mathrm{d}} \sim 4.5$ and $\tau_{\mathrm{d}} \sim 10$, respectively, providing very accurate estimates, as can be seen in Fig. 16. An estimate of the time of minimum sphericity is more difficult to deduce, but it seems to be close to $t_{\Psi \min } \sim \bar{d}_{\mathrm{cc}} / V_{\mathrm{LGK}}$, i.e. $\tau_{\Psi \text { min }} \sim \bar{d}_{\text {cc }} / l_{\text {diff. }}$ This gives $\tau_{\Psi \min } \sim 7, \tau_{\Psi \text { min }} \sim 11$ and 


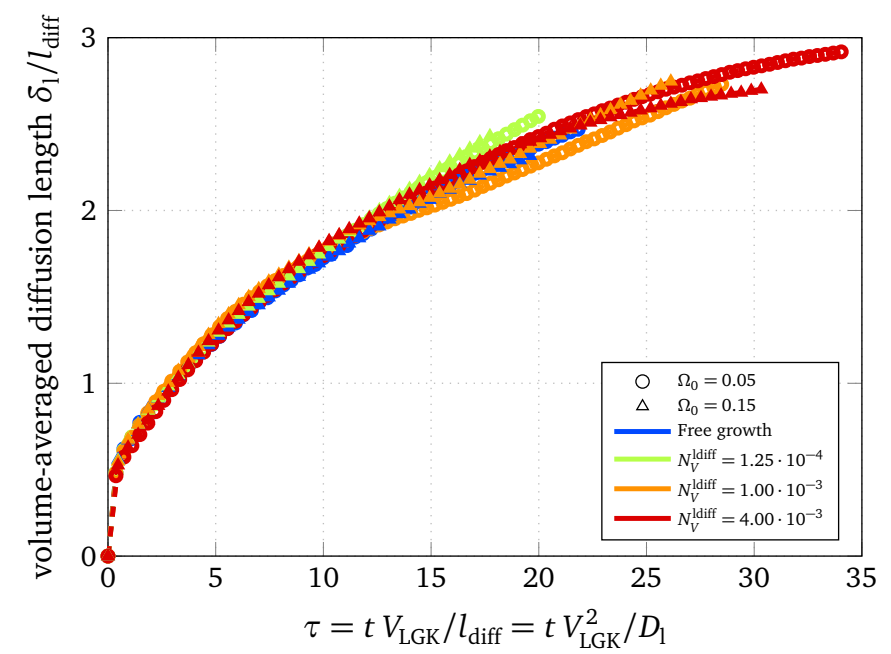

Fig. 17 Evolution of the dimensionless volume-averaged diffusion length, $\delta_{1} /\left(D_{1} / V_{\mathrm{LGK}}\right)$, over the dimensionless time.

$\tau_{\Psi \min } \sim 22$ for the three grain densities, from the highest to the smallest.

The evolution of the dimensionless average diffusion length, $\delta_{1} /\left(D_{1} / V_{\mathrm{LGK}}\right)$, with time is shown in Fig. 17. We can see that the evolution is independent of the initial supersaturation as well as of the grain density.

\section{Conclusions and outlook}

We have performed a systematic analysis of the performance of the mesoscopic envelope field model of dendritic solidification. The sensitivity of the results to the numerical parameters - the spatial discretization and the stabilization parameter of the phase-field interface capturing method was quantified in detail by extensive convergence and parameter studies. We introduced a new, more accurate method for the determination of the distance from a grid cell to the dendrite envelope, used in the matching of the analytical tip solution to the numerical solution of the mesoscopic diffusion field. This improves the accuracy of the calculation of the envelope growth velocity and thus increases the application range of the mesoscopic model.

We have performed a detailed validation of the model, focusing on the aspects that determine its main power: the fidelity of the description of solutal interactions between neighboring grains. This fidelity depends on the the accuracy of the representation of the envelope growth velocity, of the shape of the grain envelopes, and of the solute flux rejected into the liquid surrounding the grain. The validation was done by comparison of model predictions of these aspects to theoretical and experimental references. The validation procedure has shown that the model results depend on the stagnant film thickness, the only model parameter, and that an appropriate choice of this parameter is required to ensure accurate predictions. We have shown that a stagnant film thickness of the order of the theoretical primary tip diffusion length, $D_{1} / V_{\mathrm{LGK}}$, leads to accurate predictions in terms of the dynamics, the shape and the internal solid fraction of equiaxed grain envelopes. This demonstrated that the mesoscopic model gives physically realistic results for threedimensional equiaxed dendritic growth in binary alloys over a wide range of undercoolings.

From the analysis of the numerical performance and from the validation study we were able to provide generally valid guidelines for the calibration of the mesoscopic model. The knowledge of the quantitative dependence of the errors on the numerical and model parameters gives the possibility to carefully control the accuracy of model predictions.

The model has been applied to simulate the growth of large ensembles of equiaxed grains in the presence of solutal interactions. The configurations ranged from weakly to strongly interacting. To quantify the grain interactions and their dynamics and to demonstrate the possibilities of scale bridging from the mesoscopic to macroscopic models, we calculated representative volume-averaged quantities for the simulated ensembles. We have shown examples of the temporal evolution of the volume-averaged envelope surface area and of the volume-averaged diffusion length in the liquid at the envelope surface. With these examples we have shown that the proper choice of scalings (in our examples for time) can lead to generalized correlations that can be implemented in volume-averaged multiscale models of processes, which are used at the industrial scale.

Upcoming work will consist of the inclusion of melt convection in the model, in order to describe its influence on grain growth and grain interactions. In parallel, scalebridging to macroscopic models, first demonstrated in this paper, will be developed with the objective of providing refined laws and parameters for the description of microstructures in volume-averaged macroscopic models.

\section{Acknowledgement}

This work was supported by postdoctoral fellowships from the French State through the program "Investment in the future" operated by the National Research Agency (ANR) and referenced by ANR-11 LABX-0008-01 (LabEx DAMAS), and by the National Polytechnic Institute of Lorraine under the grant INPL-2011-UMR7198-M3-0052. C.B. was supported by NASA under grant NNX14AD69G. The authors would like to thank H. Jasak and H. Rusche for the help with the programming provided during the OpenFOAM Summer School 2013.

\section{References}

[1] G. P. Ivantsov, Dokl. Akad. Nauk SSSR 58 (1947) 567-570.

[2] G. Horvay, J. W. Cahn, Acta Metall. 9 (1961) 695-705.

[3] W. J. Boettinger, J. A. Warren, C. Beckermann, A. Karma, Annu. Rev. Mater. Res. 32 (2002) 163-194.

[4] I. Steinbach, JOM 65 (2013) 1096-1102.

[5] A. Choudhury, K. Reuther, E. Wesner, A. August, B. Nestler, M. Rettenmayr, Comput. Mater. Sci. 55 (2012) 263-268.

[6] T. Takaki, T. Shimokawabe, M. Ohno, A. Yamanaka, T. Aoki, J. Cryst. Growth 382 (2013) 21-25. 
[7] Z. Guo, S. M. Xiong, IOP Conf. Ser.: Mater. Sci. Eng. 84 (2015) 012067.

[8] M. Rappaz, C.-A. Gandin, Acta Metall. Mater. 41 (1993) 345-360.

[9] C.-A. Gandin, T. Carozzani, H. Digonnet, S. Chen, G. Guillemot, JOM 65 (2013) 1122-1130.

[10] J. Ni, C. Beckermann, Metall. Trans. B 22 (1991) 349-361.

[11] C. Y. Wang, C. Beckermann, Metall. Mater. Trans. A 27 (1996) 2754 2764.

[12] M. Wu, A. Fjeld, A. Ludwig, Comput. Mater. Sci. 50 (2010) 32-42.

[13] N. Leriche, H. Combeau, C.-A. Gandin, M. Založnik, IOP Conf. Ser.: Mater. Sci. Eng. 84 (2015) 012087.

[14] I. Steinbach, C. Beckermann, B. Kauerauf, Q. Li, J. Guo, Acta Mater. 47 (1999) 971-982.

[15] I. Steinbach, H.-J. Diepers, C. Beckermann, J. Cryst. Growth 275 (2005) 624-638.

[16] P. Delaleau, C. Beckermann, R. H. Mathiesen, L. Arnberg, ISIJ Int. 50 (2010) 1886-1894.

[17] M. Založnik, A. Viardin, Y. Souhar, H. Combeau, M. Apel, IOP Conf. Ser: Mater. Sci. Eng. 84 (2015) 012074.

[18] A. J. Melendez, C. Beckermann, J. Cryst. Growth 340 (2012) 175-189.

[19] Q. Li, C. Beckermann, Phys. Rev. E 57 (1998) 3176-3188.

[20] M. E. Glicksman, E. Winsa, R. C. Hahn, T. A. Lograsso, S. H. Tirmizi, M. E. Selleck, Metall. Trans. A 19 (1988) 1945-1953.

[21] M. Glicksman, R. Smith, M. Koss, L. Bushnell, J. LaCombe, in: S. Sadhal, A. Gopinath (Eds.), Heat Transf. Microgravity Syst. ASME HTDVol.290, ASME, New York, 1994, pp. 1-8.

[22] J. C. LaCombe, M. B. Koss, V. E. Fradkov, M. E. Glicksman, Phys. Rev E 52 (1995) 2778-2786.

[23] R. H. Mathiesen, L. Arnberg, Acta Mater. 53 (2005) 947-956.

[24] R. H. Mathiesen, L. Arnberg, P. Bleuet, A. Somogyi, Metall. Mater. Trans. A 37 (2006) 2515-2524.

[25] J. Lipton, M. E. Glicksman, W. Kurz, Metall. Trans. A 18 (1987) 341345.

[26] B. Cantor, A. Vogel, J. Cryst. Growth 41 (1977) 109-123.

[27] Y. Sun, C. Beckermann, J. Comput. Phys. 220 (2007) 626-653.

[28] Y. Sun, C. Beckermann, Phys. D Nonlinear Phenom. 237 (2008) 30893098.

[29] C. Beckermann, R. Viskanta, Appl. Mech. Rev. 46 (1993) 1-27.

[30] M. Založnik, H. Combeau, Comput. Mater. Sci. 48 (2010) 1-10.

[31] G. M. Treece, R. W. Prager, A. H. Gee, Comput. Graph. 23 (1998) 583598.

[32] P. J. Roache, Annu. Rev. Fluid Mech. 29 (1997) 123-160.

[33] M. B. Koss, J. C. LaCombe, L. A. Tennenhouse, M. E. Glicksman, E. A. Winsa, Metall. Mater. Trans. A 30 (1999) 3177-3190.

[34] Q. Li, C. Beckermann, J. Cryst. Growth 236 (2002) 482-498.

[35] M. Rappaz, P. H. Thévoz, Acta Metall. 35 (1987) 1487-1497.

[36] C. Y. Wang, C. Beckermann, Mater. Sci. Eng. A 171 (1993) 199-211.

[37] B. Appolaire, H. Combeau, G. Lesoult, Mater. Sci. Eng. A 487 (2008) $33-45$.

[38] H. Combeau, M. Založnik, S. Hans, P. Richy, Metall. Mater. Trans. B 40 (2009) 289-304. 